\title{
LEReC Machine Protection System
}

\author{
S. Seletskiy, Z. Altinbas, M. Costanzo, A. Fedotov, \\ D.M. Gassner, L. Hammons, J. Hock, P. Inacker, \\ J. Jamilkowski, D. Kayran, K. Mernick, T. Miller, M. Minty, \\ M. Paniccia, W. Pekrul, I. Pinayev, K. Smith, Y. Than, \\ P. Thieberger, J. Tuozzolo, W. Xu, Z. Zhao
}

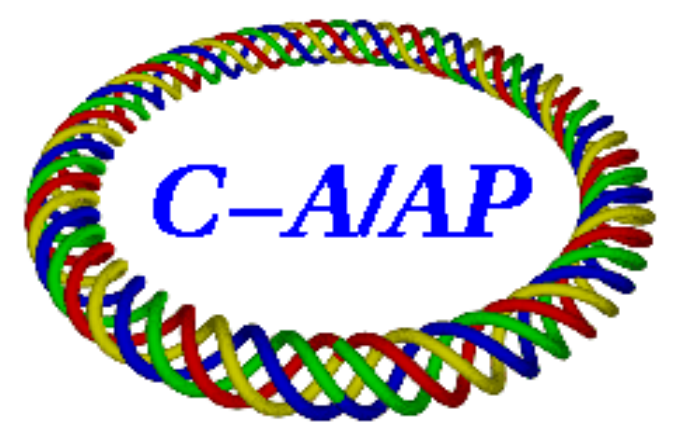

\section{Collider-Accelerator Department Brookhaven National Laboratory Upton, NY 11973}

\section{U.S. Department of Energy}

Office of Science, Office of Nuclear Physics

Notice: This document has been authorized by employees of Brookhaven Science Associates, LLC under Contract No. DE-SC0012704 with the U.S. Department of Energy. The United States Government retains a nonexclusive, paid-up, irrevocable, world-wide license to publish or reproduce the published form of this document, or allow others to do so, for United States Government purposes. 


\section{LEReC Machine Protection System}

S. Seletskiy, Z. Altinbas, M. Costanzo, A. Fedotov, D. M. Gassner, L. Hammons, J. Hock, P. Inacker, J. Jamilkowski, D. Kayran, K. Mernick, T. Miller, M. Minty, M. Paniccia, W. Pekrul, I. Pinayev, K. Smith, Y. Than, P. Thieberger, J. Tuozzolo, W. Xu, Z. Zhao

April 14, 2017

\section{Contents}

1. Introduction

1.1 LEReC beam parameters

1.2 LEReC beam modes

2. MPS parameters

2.1 MPS reaction time

2.2 Tolerable routine losses

2.3 Ultimately safe operation mode

2.4 MPS parameters summary

3. Failure scenarios

3.1 Beam loss inside or near the gun

3.2 Beam loss inside the SRF Booster

3.3 Wrong power beam hits in-vacuum component

3.4 Summary of failure scenarios

4. MPS diagnostics

4.1 List of MPS equipment

4.2 LEReC FCT

5. Laser - MPS interface

6. Gun - MPS interface

7. MPS logic

7.1 MPS schematic

7.2 Concept of MPS logic

7.3 MPS logic

8. Commissioning procedures

8.1 Integrated system test without beam (for 2017 run)

8.2 MPS commissioning with beam (for 2017 run)

9. Operation procedures

9.1 Routine MPS test

9.2 Verification of MPS integrity

10. Conclusion 


\section{Introduction}

The LEReC Machine Protection System (MPS) is designed to alleviate the risk of possible damage to in-vacuum components of the accelerator from high power electron beam.

\subsection{LEReC beam parameters}

The LEReC accelerator consists of the $400 \mathrm{keV}$ DC photo-gun followed by the 1.6-2.2 MeV SRF Booster, the transport line, the merger that brings the beam to the two cooling sections (CS1 and CS2) and the cooling sections followed by the $140 \mathrm{~kW}$ dump. The LEReC also includes two dedicated diagnostic beamlines: the low-power beamline capable of accepting $10 \mathrm{~kW}$ beam and the RF diagnostic beamline. The LEReC layout is schematically shown in Fig. 1.

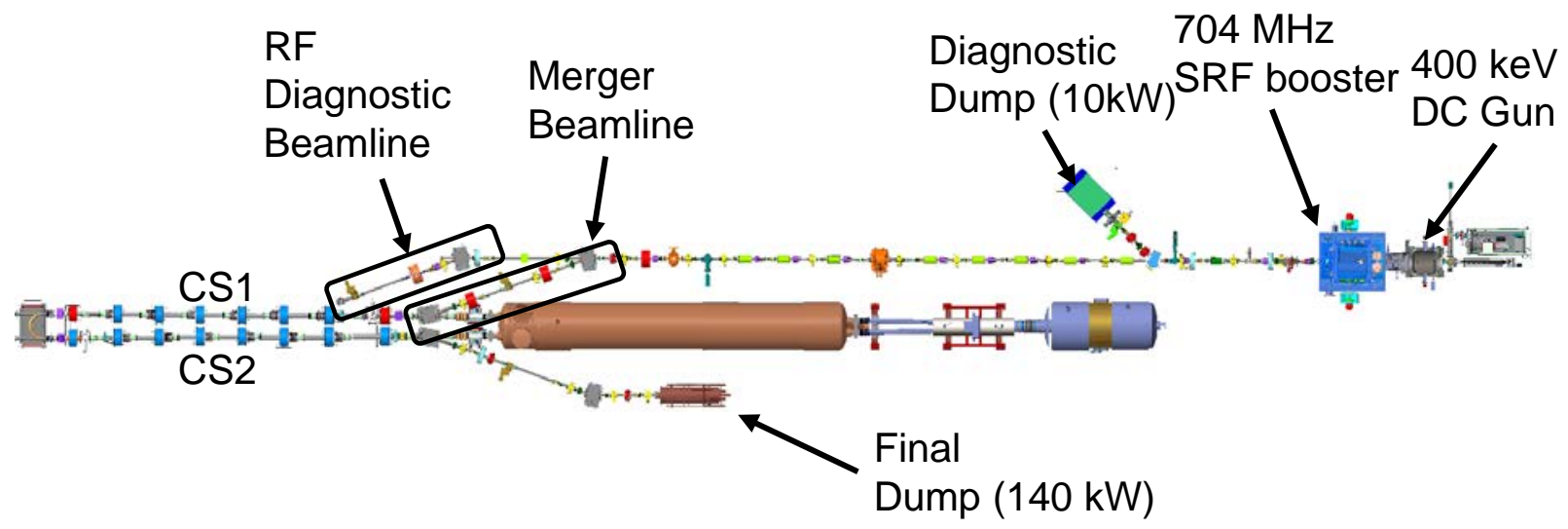

Figure 1: The LEReC layout.

The LEReC operates with electron beam consisting of $9 \mathrm{MHz}$ macrobunches. Each macrobunch consists of up to thirty $704 \mathrm{MHz}$ bunches. The nominal beam parameters of the LEReC are summarized in Table 1.

\begin{tabular}{|l|c|c|c|}
\hline Kinetic Energy, MeV & 1.6 & 2 & 2.6 \\
\hline Electron bunch $(704 \mathrm{MHz})$ charge, $\mathrm{pC}$ & 130 & 170 & 200 \\
\hline Bunches per macrobunch $(9 \mathrm{MHz})$ & 30 & 30 & $24-30$ \\
\hline Charge per macrobunch, $\mathrm{nC}$ & 4 & 5 & $5-6$ \\
\hline Average current, $\mathrm{mA}$ & 35 & 46 & $44-55$ \\
\hline Average power, $\mathrm{kW}$ & 56 & 93 & $114-142$ \\
\hline
\end{tabular}

Table 1: The LEReC beam parameters pertinent to the MPS design.

In addition to baseline operational modes listed in Table 1 the LEReC might also be operated with CW $704 \mathrm{MHz}$ beam of $85 \mathrm{~mA}$ (at $1.6 \mathrm{MeV}$ ) and $68 \mathrm{~mA}$ (at $2 \mathrm{MeV}$ ). Such beam has $136 \mathrm{~kW}$ average power.

The maximum beam energy that can be inadvertently achieved in the LEReC is $3 \mathrm{MeV}$. 


\subsection{LEReC beam modes}

In addition to nominal operation beam modes outlined in Section 1.1 there are several additional beam modes required for accelerator commissioning, study and transition to operational conditions. All required LEReC beam modes are described in Table 2 and are schematically represented in Fig. 2 .

\begin{tabular}{|c|c|c|c|}
\hline Timing Pattern & Beam modes & Goals & Power \& Current \\
\hline $\begin{array}{l}N_{\mathrm{b}}=30 \\
N_{\mathrm{mb}}=1 \\
T=1 \mathrm{~s}\end{array}$ & $\begin{array}{l}\text { Low Current Mode } \\
(\text { LCM); } \\
Q_{b}=30-200 p C\end{array}$ & $\begin{array}{l}\text { Obtain beam trajectory through } \\
\text { accelerator. First iteration on setting } \\
\text { the RF. Set optics for nominal } Q_{b} \text {. } \\
\text { measure beam emittance. Measure } \\
\text { beam envelope in the CS. }\end{array}$ & $\begin{array}{l}P \leq 16 \mathrm{~mW} \\
\mathrm{I} \leq 6 \mathrm{nA}\end{array}$ \\
\hline $\begin{array}{l}N_{\mathrm{b}}=10,15,20,25,30 \\
\Delta t \leq 250 \text { us } \\
T=1 \mathrm{~s}-5 \mathrm{~s}\end{array}$ & $\begin{array}{l}\text { RF Studies Mode } \\
\text { (RFSM); } \\
Q_{\mathrm{b}} \leq 200 \mathrm{pC}\end{array}$ & $\begin{array}{l}\text { RF fine-tuning. Study beam } \\
\text { longitudinal phase space. }\end{array}$ & $\begin{array}{l}P \leq 7 W \\
I \leq 3 \text { uA }\end{array}$ \\
\hline \multirow{2}{*}{$\begin{array}{l}N_{\mathrm{b}}=30 \\
\Delta t \leq 1000 \mathrm{~ms} \\
T=1 \mathrm{~s}\end{array}$} & $\begin{array}{l}\text { Transition Mode } 1 \\
\text { (TM1); } \\
Q_{\mathrm{b}}=200 \mathrm{pC}\end{array}$ & $\begin{array}{l}\text { Gradual transition from LCM to HCM } \\
\text { keeping nominal } Q_{\mathrm{b}} \text {. }\end{array}$ & $\begin{array}{l}\mathrm{P} \leq 142 \mathrm{~kW} \\
\mathrm{I} \leq 55 \mathrm{~mA}\end{array}$ \\
\hline & $\begin{array}{l}\text { Transition Mode } 2 \\
(\mathrm{TM} 2) ; \\
Q_{\mathrm{b}} \leq 200 \mathrm{pC}\end{array}$ & $\begin{array}{l}\text { Gradual transition from } \mathrm{LCM} \text { to } \mathrm{HCM} \\
\text { with continuous } 9 \mathrm{MHz} \text { train of } \\
\text { macrobunches and the gradual } \\
\text { adjustment of } Q_{\mathrm{b}} \text {. }\end{array}$ & $\begin{array}{l}\mathrm{P} \leq 142 \mathrm{~kW} \\
\mathrm{I} \leq 55 \mathrm{~mA}\end{array}$ \\
\hline $\begin{array}{l}N_{\mathrm{b}}=30 \\
\Delta t=T\end{array}$ & $\begin{array}{l}\text { High current Mode } \\
(\mathrm{HCM}) ; \\
Q_{\mathrm{b}}=130-200 \mathrm{pC}\end{array}$ & $\begin{array}{l}\text { Getting nominal e-beam parameters } \\
\text { in the CS. }\end{array}$ & $\begin{array}{l}P=56-142 \mathrm{~kW} \\
\mathrm{I}=35-55 \mathrm{~mA}\end{array}$ \\
\hline $704 \mathrm{MHz} C W$ & $\begin{array}{l}\text { CW Mode (CWM); } \\
Q_{\mathrm{b}}=95-120 \mathrm{pC}\end{array}$ & Alternative to $\mathrm{HCM}$. & $\begin{array}{l}P=136 \mathrm{~kW} \\
\mathrm{I}=68-85 \mathrm{~mA}\end{array}$ \\
\hline
\end{tabular}

Table 2: The LEReC beam modes. 
$\mathrm{Q}_{\mathrm{b}}$ - charge per bunch (operational $130 \mathrm{pC}$ )

$\mathrm{N}_{\mathrm{b}}$ - number of bunches per macro-bunch (operational 30)

$\Delta \mathrm{t}$ - length of train of macro-bunches

$\mathrm{N}_{\mathrm{mb}}$ - number of macro-bunches per train

$\mathrm{T}$ - time period between trains

(a)
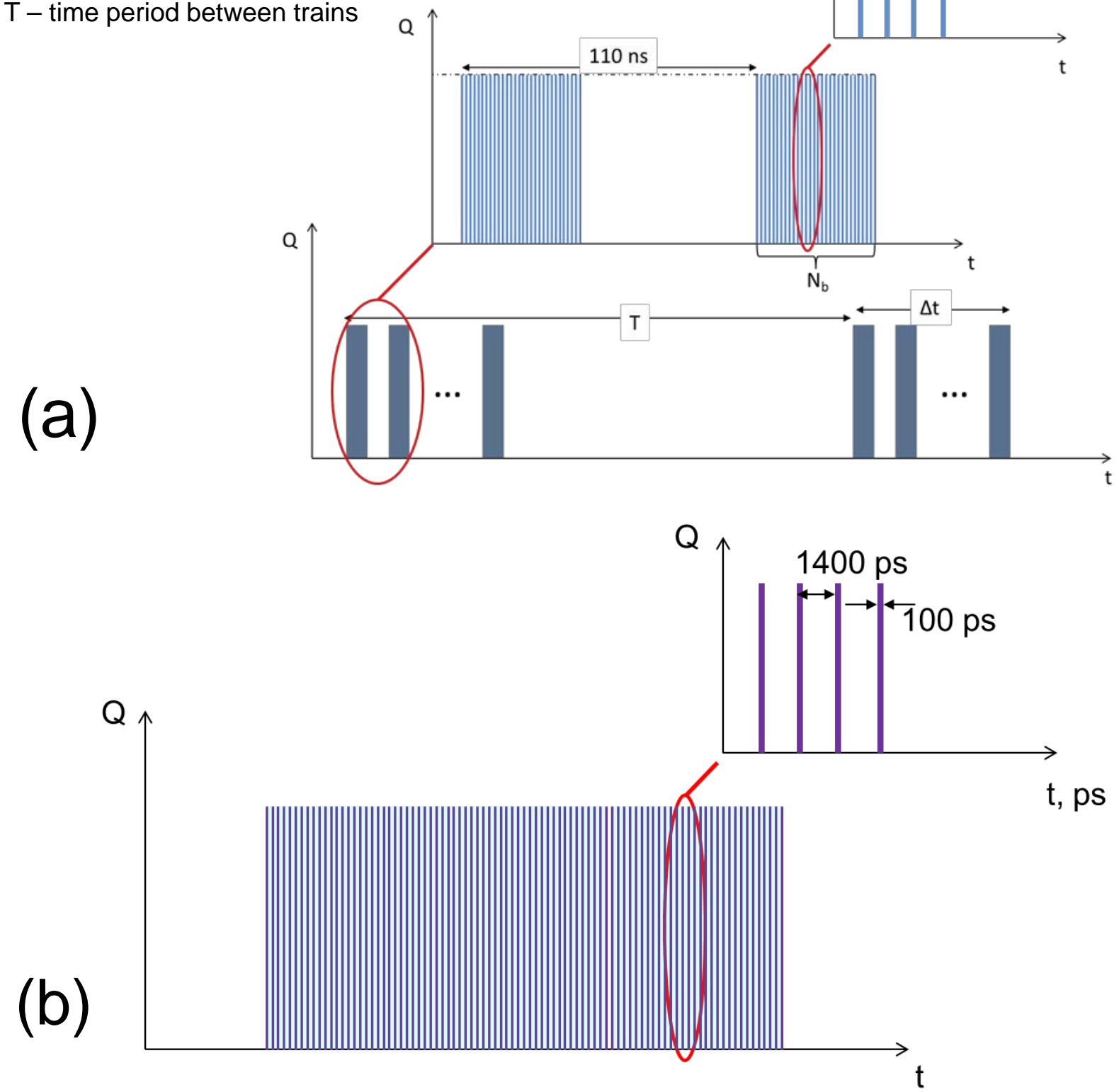

Figure 2: The LEReC beam modes. Figure (a) corresponds to a continuous sequence of $9 \mathrm{MHz}$ macrobunches or trains (of length $\Delta t$ ) of $9 \mathrm{MHz}$ macrobunches repeated with frequency $1 / T$. Figure (b) represents either $\mathrm{CW} 704 \mathrm{MHz}$ bunches or trains of length $\Delta t_{\mathrm{CW}}$ of $704 \mathrm{MHz}$ bunches. 


\section{MPS parameters}

\subsection{MPS reaction time}

The MPS reaction time must be less than the time required for the high power beam to damage the in-vacuum components.

The highest energy that can be inadvertently achieved with the planned LEReC RF system is $3 \mathrm{MeV}$. The typical transverse rms beam size $(\sigma)$ in the LEReC is larger than $1 \mathrm{~mm}$ ( $2 \mathrm{~mm}$ on average) with the exclusion of three locations in the merger beamline where it is focused down to $\sigma=0.25 \mathrm{~mm}$ (Fig. 3).

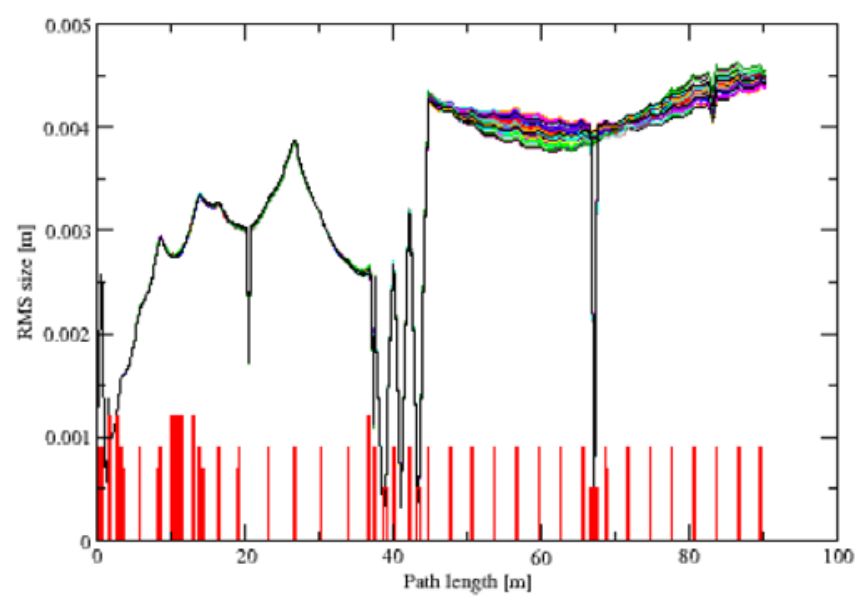

Figure 3: Beam transverse rms beam size throughout the LEReC.

The thickness of the LEReC vacuum chamber is $w=2 \mathrm{~mm}$ so it stops the $100 \%$ of the electron beam. The stainless steel 304 melting temperature is $1450 \mathrm{C}$ and the stainless steel temperature to reach ultimate yield strength is $170 \mathrm{C}$. Therefore, according to (1), if the tightly focused CW e-beam (85 mA current) with $3 \mathrm{MeV}$ energy hits vacuum chamber at normal incident angle then it takes just 1.3 us and 13 us to reach the maximum yield strength or to melt the vacuum chamber respectively.

$$
\Delta T=\frac{P \cdot t}{S H C \cdot \rho \cdot \pi(\sqrt{2} \sigma)^{2} w}
$$

Here, $T$ is the temperature increase in time $t, P$ is the beam power, the stainless steel specific heat capacity $(S H C)$ is $502.4 \mathrm{~J} /(\mathrm{kg} \cdot \mathrm{K})$ and its density $\rho=7999.5 \mathrm{~kg} / \mathrm{m}^{3}$.

The only locations where the beam can have $90^{\circ}$ incident angle are the $\mathrm{Y}$-shaped dipole chambers (Fig. 1, Fig. 4). 


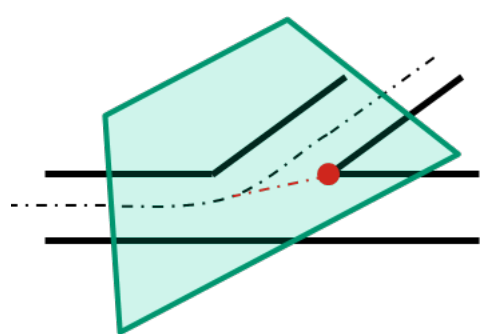

Figure 4: Beam trajectory through the dipole. Red dashed line shows the missteered beam hitting the crotch of the dipole chamber.

The beam can be missteered by the magnets. Since the changes in magnets currents and fields are relatively slow we can protect against such an event by locking the respective magnet power supply currents within some reasonable window.

Another possibility for beam missteering is the RF phase jump, which can happen within a few microseconds. Fortunately, due to the geometry of our machine the beam energy must be increased for the beam to hit the crotch of the dipole chamber and there is simply not enough RF power to increase the beam energy by the required value.

Therefore, the high current tightly focused beam can hit the vacuum chamber only at some angle. We define the maximum possible angle to be $35 \mathrm{mrad}$ from the vacuum chamber diameter and the minimal distance between the adjacent magnets in the merger beamline. Next, we assume that the large $(\sigma=1 \mathrm{~mm})$ size beam can hit such in-vacuum components as vacuum valves or flag mirrors at normal incident angle in case they are inserted by mistake during high current operation. We also assume that all beam trajectory and focusing studies are performed at LCM and the beam trajectory is getting locked in BPMs within some reasonable window for HCM.

We performed a set of calculations, both with (1) and with ANSYS (see Fig. 5 for an example), under the aforementioned assumptions. The results of analytical estimates and ANSYS modeling well agree due to the fact that very little thermalization is happening within the times characteristic to considered process. The results of these studies are summarized in Table 3. 

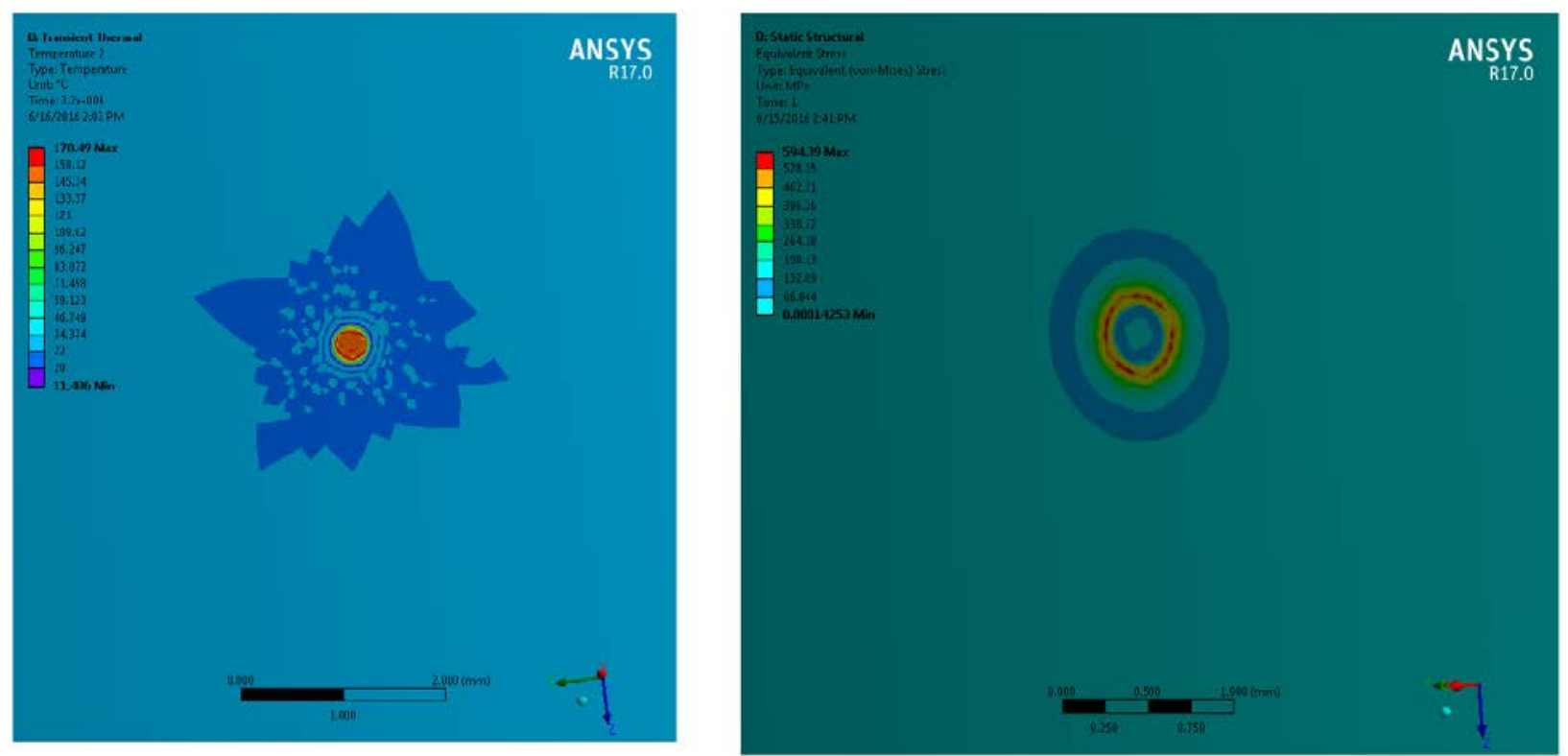

Figure 5: The results of ANSYS simulations for $110 \mathrm{~kW}$ beam with $\sigma=0.25 \mathrm{~mm}$ hitting the vacuum chamber at normal incident angle. The left plot shows temperature distribution in $t=3.4$ us and the right plot shows the thermal stress at $170 \mathrm{C}$ (the stainless steel ultimate yield strength is $590 \mathrm{Mpa}$ ).

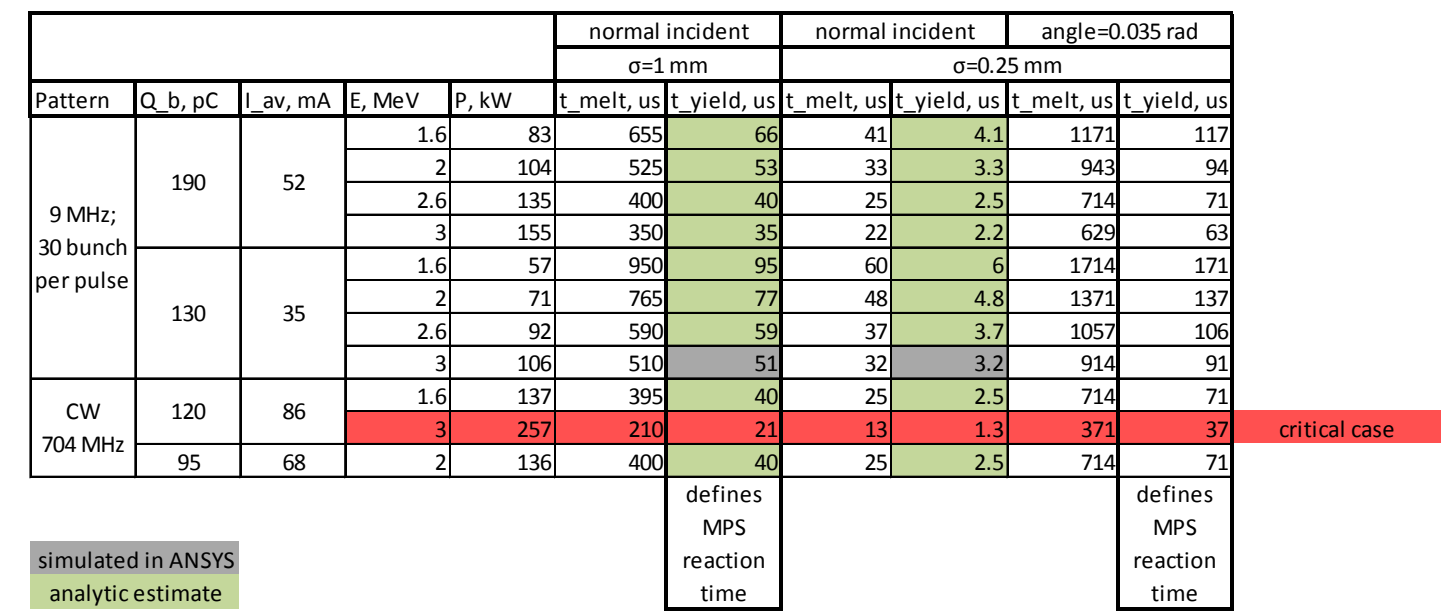

Table 3: Results of studies of the thermal effect that the lost e-beam has on the in-vacuum components.

From the studies summarized in Table 3 we require that the MPS reaction time $\left(t_{\text {react }}\right)$ is 20 us.

\section{Section 2.1 summary:}

- Required MPS reaction time is 20 us

- All beam trajectory and focusing studies are performed in LCM only

- In HCM the beam trajectory is locked in BPMs within some reasonable window and some magnet (dipole, for sure) power supply currents are locked within some reasonable window. 


\subsection{Tolerable routine losses}

Apparently there is some loss current that can be tolerated during routine machine operations. The threshold for tolerable losses will define the requirements to the precision of beam loss diagnostics.

The routine operational losses will be due to the beam halo and eventually the threshold for the tolerable loss will be determined experimentally. Nonetheless, to have a starting point for the initial MPS setup we are going to intentionally underestimate this threshold by assuming that the loss is caused by the whole beam (not a tightly focused one) hitting the vacuum chamber at some incident angle.

The typical size of the beam throughout the LEReC is $\sigma=2 \mathrm{~mm}$, which corresponds to the equivalent uniform density circle of radius $R_{0} \approx 2.83 \mathrm{~mm}$.

The worst incident angle ( $\alpha=42 \mathrm{mrad}$ ) is defined by the distance between the magnets in the cooling section and the diameter of the CS vacuum chamber (Fig. 6). It's worth noticing that in the cooling section the beam size is $\sigma=4 \mathrm{~mm}$, so here we are again intentionally tightening our initial loss threshold.

$$
\alpha<\frac{5 \cdot 2.54 \cdot 10^{-2}}{3}=0.042 \mathrm{rad}
$$

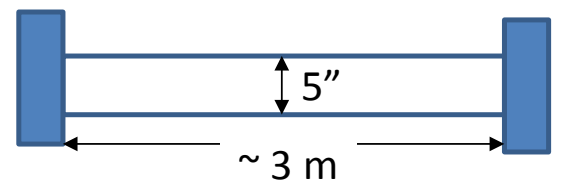

Figure 6: The worst incident angle is derived from the CS vacuum chamber dimensions and distance between the CS solenoids.

Next, we assume that the power of the lost beam is transferred away from the hit location through conductance only. For stainless steel 304 thermal conductivity is $\kappa=16.2 \mathrm{~W} / \mathrm{m} \cdot \mathrm{K}$.

The beam spot on the vacuum chamber can be approximated as an ellipse with semi-axes $a=R_{0} / \tan \alpha$ and $b=R_{0}$. To simplify calculations we are substituting an ellipse with a circle of the same circumference. Such a circle has a radius $R_{1} \approx \frac{2 R_{0}}{\pi \cdot \tan \alpha} \cdot \xi(e)$, where $\xi$ is the complete elliptic integral of the second kind and $e=\frac{\sqrt{\cos (2 \alpha)}}{\cos \alpha}$ is the ellipse eccentricity.

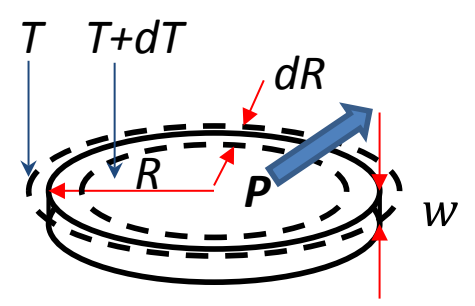

Figure 7: The model of the power transfer in steady state.

The equation for power transfer in steady state (illustrated in Fig. 7) is given by (2):

$$
P=-\kappa 2 \pi R w \frac{d T}{d R}
$$


Solution of (2) is given by:

$$
P=\frac{\kappa T 2 \pi w}{-\ln R}
$$

Apparently, the highest temperature will be reached at the spot of the beam hit. Therefore, substituting $R_{1}$ into (3) we obtain that depending on the maximum temperature increase of the vacuum chamber that one allows $\left(T_{\max }\right)$, the allowed power $\left(P_{\text {loss }}\right)$ of the routine loss is:

$$
P_{\text {loss }}=\frac{\kappa T_{\max } 2 \pi w}{-\ln \left(\frac{2 R_{0}}{\pi \cdot \tan \alpha} \cdot \xi\left(\frac{\sqrt{\cos (2 \alpha)}}{\cos \alpha}\right)\right)}
$$

Assuming $T_{\max }=50 \mathrm{~K}$ we get $P_{\text {loss }}=3 \mathrm{~W}$, which for $3 \mathrm{MeV}$ beam corresponds to the threshold on the allowed loss current of $I_{\text {loss }}=1 \mathrm{uA}$.

As we mentioned before, this small current will be our starting point in empirical search for the tolerable routine current losses. To find such losses experimentally we will intentionally generate the loss of defocused small current beam and observe the vacuum activity at the location of the loss.

It is important to define the maximum current that we may use in the described studies. The reaction time of the LEReC vacuum system is on the order of $100 \mathrm{~ms}$. Therefore, the maximum current used for the studies must not damage the vacuum chamber in $100 \mathrm{~ms}$. If we allow the vacuum temperature rise of $50 \mathrm{~K}$ then according to (1) the current of $3 \mathrm{MeV} \mathrm{CW}$ beam with $\sigma=2 \mathrm{~mm}$ must not exceed $I_{\max }=600 \mathrm{uA}$.

\section{Section 2.2 summary:}

- The initial setting for the tolerable routine losses is $I_{\text {loss }}=1 \mathrm{uA}$.

- The eventual setting of tolerable loss threshold will be found experimentally by generating the loss of the defocused beam and observing the vacuum activity at the location of the loss. The beam current used for these studies must not exceed $I_{\max }=600 \mathrm{uA}$.

\subsection{Ultimately safe operation mode}

In an ultimately safe operation mode (USOM) we allow any manipulations with the electron beam. To set the current threshold for the USOM ( Usom$_{\text {U }}$ ) we consider the most extreme conditions possible. Let us assume the beam energy of $3 \mathrm{MeV}$, transverse beam size of $0.25 \mathrm{~mm}$, normal incident angle, $200 \mathrm{pC}$ per bunch and $704 \mathrm{MHz} \mathrm{CW}$ operation mode. If we allow only $50 \mathrm{~K}$ temperature increase for the hit spot then according to (1) we can afford only 0.28 us beam trains and assuming $1 \mathrm{~Hz}$ repetition rate we get $I_{\text {USOM }}=40 \mathrm{nA}$. 
Apparently, the LCM is the USOM since the average current in LCM is $\leq 6 \mathrm{nA}$. Indeed, in the LCM under the worst case scenario $\left(N_{\mathrm{b}}=30, N_{\mathrm{mb}}=1, T=1 \mathrm{~s}, Q_{\mathrm{b}}=200 \mathrm{pC}\right)$ the vacuum chamber will be heated up by $\sim 7 \mathrm{~K}$ by an "energy hammer" hitting the chamber with $1 \mathrm{~Hz}$ rate (see Fig. 8 ).

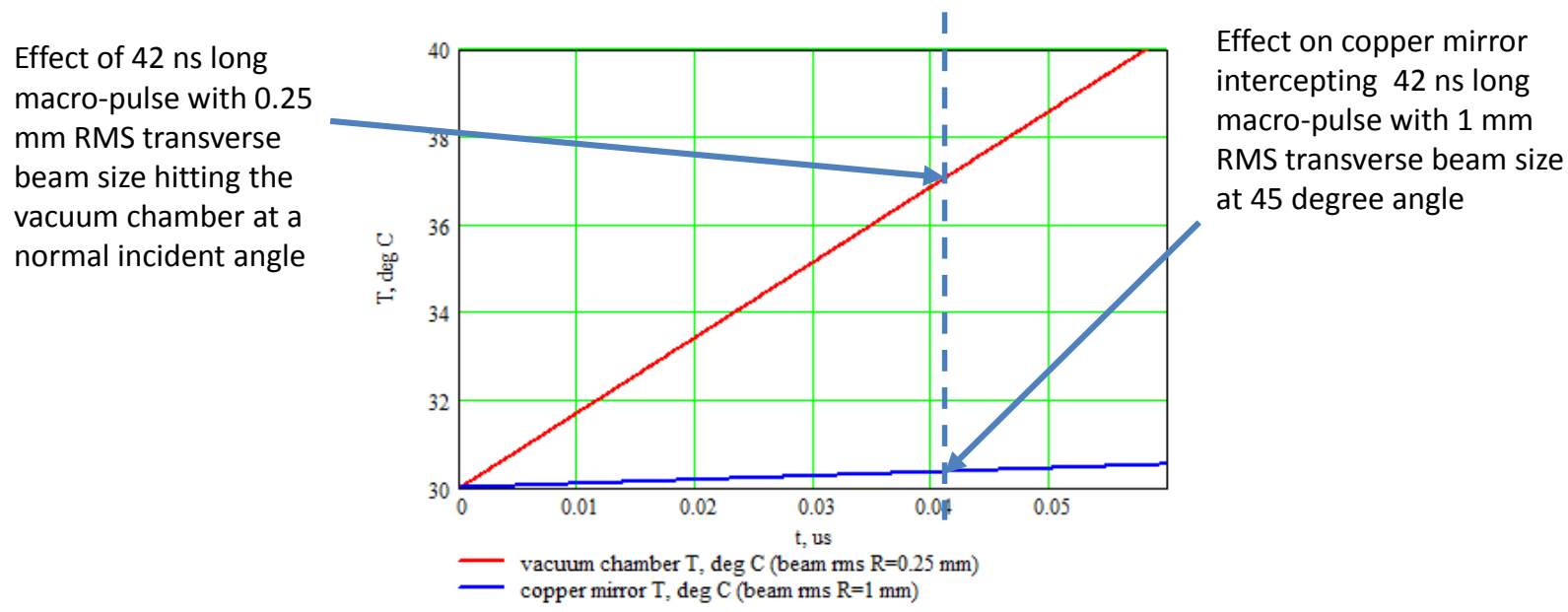

Figure 8: Expected temperature increase in the LCM due to the direct beam hit.

For stainless steel the temperature of the maximum yield strength is $170 \mathrm{C}$. Therefore the discussed "energy hammer" effect is well within the range of elastic deformation of the stainless steel and we don't expect any fatigue failure from such a small thermo-mechanical stress.

It is also worth noticing that the trains of $9 \mathrm{MHz}$ macrobunches with 30 bunches per macrobunch and $200 \mathrm{pC} /$ bunch charge repeated once a second are in the USOM as long as number of macrobunches is less than or equal to 6 .

\section{Section 2.3 summary:}

- The current threshold for the ultimately safe mode is $I_{\text {UsOM }}=40 \mathrm{nA}$.

- The LCM is the USOM.

- $\quad$ The transition mode 1 is the USOM if $N_{\mathrm{mb}} \leq 6$.

\subsection{MPS parameters summary}

Table 4 summarizes the MPS parameters. 


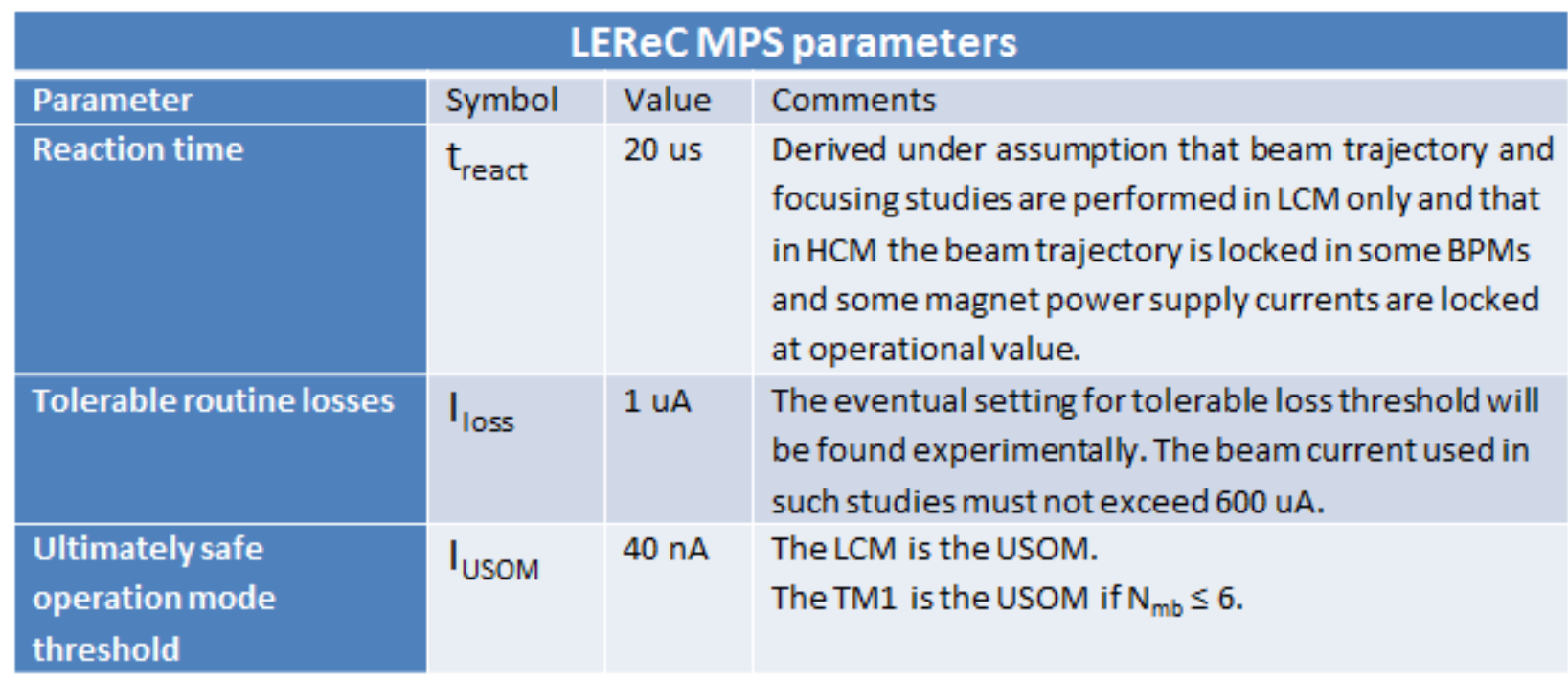

Table 4: Summary of the main MPS parameters.

\section{Failure scenarios}

The possible LEReC failure scenarios include:

- Beam loss inside or near the gun due to the incorrect settings of the anode corrector or correctors and solenoids near the gun. Another possibility for the beam loss is that it can get reflected from the SRF Booster set to the wrong phase.

- Beam loss inside the SRF Booster can be caused by the wrong beam trajectory or improper focusing. Also, there is the laser failure that can result in the train of electron bunches having the same average beam power but carrying a charge per bunch which differs from the design one. These wrong-charge bunches will not be focused properly and will get lost at the entrance of the SRF Booster.

- Finally, the wrong power beam can hit any of the following in-vacuum components: YAG screens, vacuum valves, halo monitors, emittance slits, dump or vacuum chamber.

Below we consider each of these failures in more details.

\subsection{Beam loss inside or near the gun}

The gun and diagnostics layout is schematically shown in Fig. 9. The laser from the laser room is first delivered to the in-tunnel laser table and then to the photocathode. The calibrated photodiode (PD) on the laser table measures laser power. The FCT is installed right downstream of the vacuum chamber assembly for the initial gun test and might be moved downstream of the SRF Booster for the full LEReC commissioning, although we hope to move it upstream closer to the gun if the gun-to-booster chamber gets redesigned. The beam diagnostic includes 2 BPMs for beam trajectory measurement and the insertable YAG profile monitor that will be used to guarantee proper beam size at the entrance to the SRF Booster. 


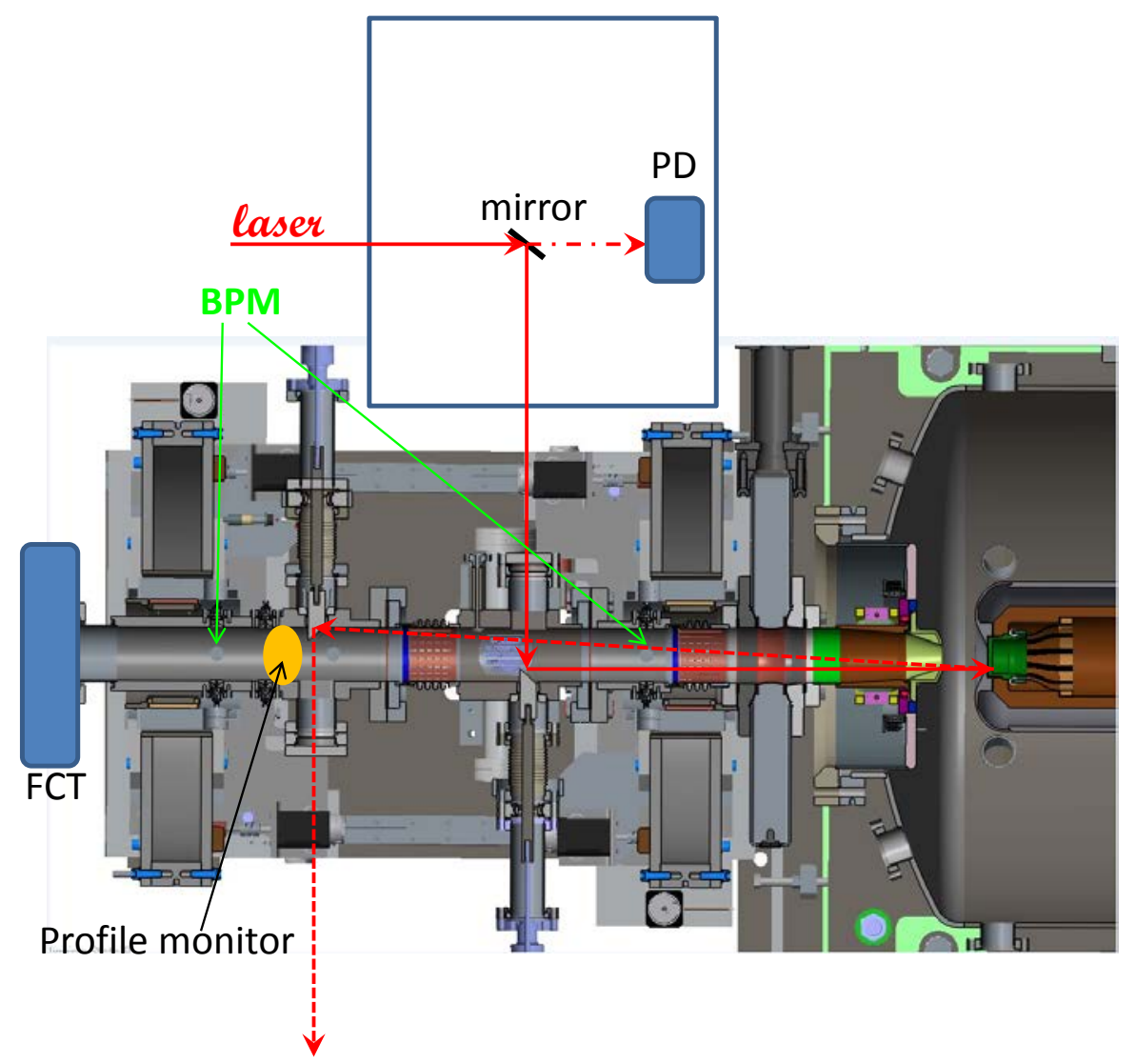

Figure 9: Schematic layout of the gun and gun exit chamber assembly.

The beam losses inside or near the gun can cause a cathode damage resulting in an unacceptable down-time. Thus, we would like to exclude such losses altogether.

We plan several lines of defense against the beam losses near or inside the gun:

- First and maybe the most important control is an administrative one. We shall start the work towards any particular charge/bunch in the LCM with minimally observable charge first. After the beam trajectory out of the gun and through the Booster is established we shall set the nominal charge/bunch (still in the LCM) and adjust the beam focusing to minimize the potential beam loss. Finally, the phase and amplitude of the SRF Booster shall be set in the LCM. Only then we may proceed with increasing the beam current.

- For modes other than LCM we shall lock the correctors (anode corrector and 2 solenoid correctors) at their optimal values. We also must lock the solenoids at the optimal values, lock beam trajectory within a few millimeter window around its optimal setting and lock the SRF Booster phase and amplitude around their optimal values.

- To monitor the losses inside and near the gun we will use the radiation beam loss monitors (BLMs), the vacuum gauges and the differential signal between the FCT and the PDmeasured power multiplied by the most recently measured cathode quantum efficiency (QE). 
The last bullet requires a few clarifications. The BLM work at LEReC beam energy has not been well tested and will require in situ calibration. This leaves us with a combination of the vacuum activity and the FCT-PD differential measurement as a distinctive characteristic of the beam loss in the gun region.

The typical reaction time of the in-gun vacuum gauges is $40 \mathrm{~ms}$. To set the requirement to the FCTPD measurement precision we assume that $400 \mathrm{keV}$ flat-top beam with $1 \mathrm{~mm}$ radius hits the in-vacuum surface at a normal incident angle. We allow the surface to be heated by less than $50 \mathrm{~K}$ in vacuum reaction time of $40 \mathrm{~ms}$. According to (1) we can allow $80 \mathrm{uA}$ loss.

Therefore, the precision of the FCT-PD measurement shall be better than $80 \mathrm{uA}$. Assuming the precision of the FCT and PD measurements to be about $1 \mathrm{pC} /$ bunch (see Section 4 for more details) we deduce that the combination of the FCT-PD and vacuum activity measurements is useful for the TM1 with up to $30 \%$ duty cycle.

\subsection{Beam loss inside the SRF Booster}

The cryogenic system of the SRF Booster allows uninterrupted operation with a few watts of continuous distributed beam losses inside the Booster. The SRF quench protection is adequate for the higher distributed losses. It is essential to protect the Booster from direct beam hit. Therefore it is critical that the steps outlined in Section 3.1 are followed religiously.

There is a highly improbable but physically viable possibility of the laser failure when the Pulse Picker (Pockels Cell working as a $9 \mathrm{MHz}$ modulator) falls in an "open" state. Such failure will result in producing the $\mathrm{CW}$ beam, which will carry the same current as continuous $9 \mathrm{MHz}$ train of macrobunches, since the laser amplifiers downstream of the Pulse Picker are already saturated, but a different charge per bunch (see Section 5 for laser schematics).

Under this scenario the $\mathrm{CW}$ e-bunches will have a mismatched charge and focusing and will get lost at the entrance of the SRF Booster where a $10 \mathrm{~cm}$ long niobium choke with $3 \mathrm{~cm}$ ID is installed.

To estimate the adequacy of the quench protection for such failure we performed the detailed studies of the extreme beam loss.

We assumed the loss power of $110 \mathrm{~kW}$ uniformly distributed over the choke surface. Taking into account that the mass of the Niobium choke area cylinder is $0.25 \mathrm{~kg}$, volume of the liquid helium in the end bell is 0.54 liter and Helium mass is $0.079 \mathrm{~kg}$ we obtained a conservative estimate of 250 us for the time required to start vaporizing helium. This time is much longer than the typical quench protection reaction time. Hence, the quench protection is adequate for the described laser failure.

\subsection{Wrong power beam hits in-vacuum component}

Any of the insertion devices, such as YAG flags, vacuum valves, halo monitors or emittance slits, can be operated in the LCM. For beam currents exceeding the USOM threshold the MPS shall stop the beam within 20 us if the insertion device position is "in".

To protect the $10 \mathrm{~kW}$ dump from the high power beam we will request the MPS to stop the beam if the beam power is higher than $10 \mathrm{~kW}$ and the respective dipole current is not zero.

To protect the vacuum chamber from the damage we will define the optimal beam trajectory and focusing in the LCM. Then we will lock the beam trajectories in BPMs in and near the merger region (see 
Section 3.1) for beam current exceeding $I_{\text {usom. }}$ We also will lock the merger dipole currents at their nominal values.

Finally, we will monitor the vacuum activity throughout the whole beamline and detect beam losses with the strategically located BLMs. For the beam losses larger than $I_{\text {loss }}$ the MPS will stop the beam within 20 us.

We will also experimentally evaluate the feasibility of using differential signal between the first FCT and FCTs installed near the dumps for detection of beam losses. If such detection is reliable we will use differential FCT signal in parallel to the BLM loss detection.

\subsection{Summary of failure scenarios}

To protect the LEReC against possible failures the following precautionary measures are suggested:

1. The work with the new beam parameters shall always start in the LCM. The beam trajectory, focusing and proper RF settings must be found in the LCM.

2. The MPS must stop the beam if the FCT-PD signal exceeds $80 \mathrm{uA}$.

3. When beam current exceeds / Usom the MPS must:

a. lock beam trajectory in certain BPMs around the value found in step 1,

b. lock certain magnets at the optimal values found in step 1 ,

c. monitor BLMs (and probably differential FCT signal) and stop the beam if loss current exceeds $I_{\text {loss }}$,

d. monitor the vacuum activity.

4. The MPS must stop the beam if any of the insertion devises are in when the beam current exceeds /usom.

\section{MPS diagnostics}

\subsection{List of MPS equipment}

The location, number and type of BLMs used for the LEReC MPS is schematically shown in Fig. 10. 


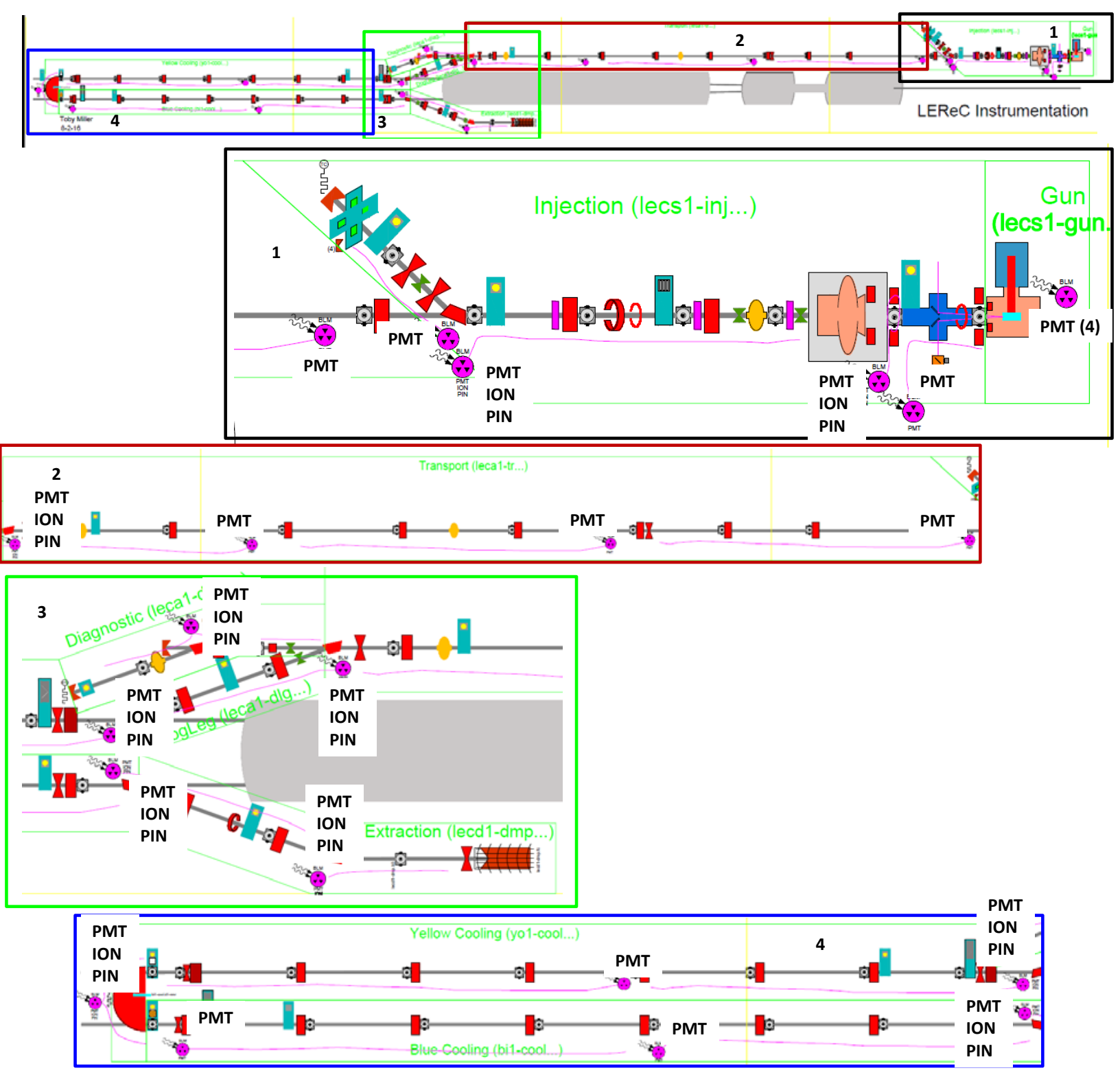

Figure 10: Layout of LEReC BLMs.

The BLMs will be calibrated with the intentional beam losses early in the gun test run. Since the calibration of BLMs can be done with defocused beam we suggest that the considerations for determining the routine loss threshold (see Section 2.2) apply to these studies. Therefore, the losses created in the dedicated studies of the BLM response shall not exceed $I_{\max }=600 \mathrm{uA}$.

There are two types of BPMs in LEReC, Libera boxes with the readout frequency of $200 \mathrm{~Hz}$ and the fast V301 boxes with response time of about 12 us. The MPS will be locking the beam trajectory in V301 type BPMs. The location of LEReC BPMs is shown in Fig. 11. 


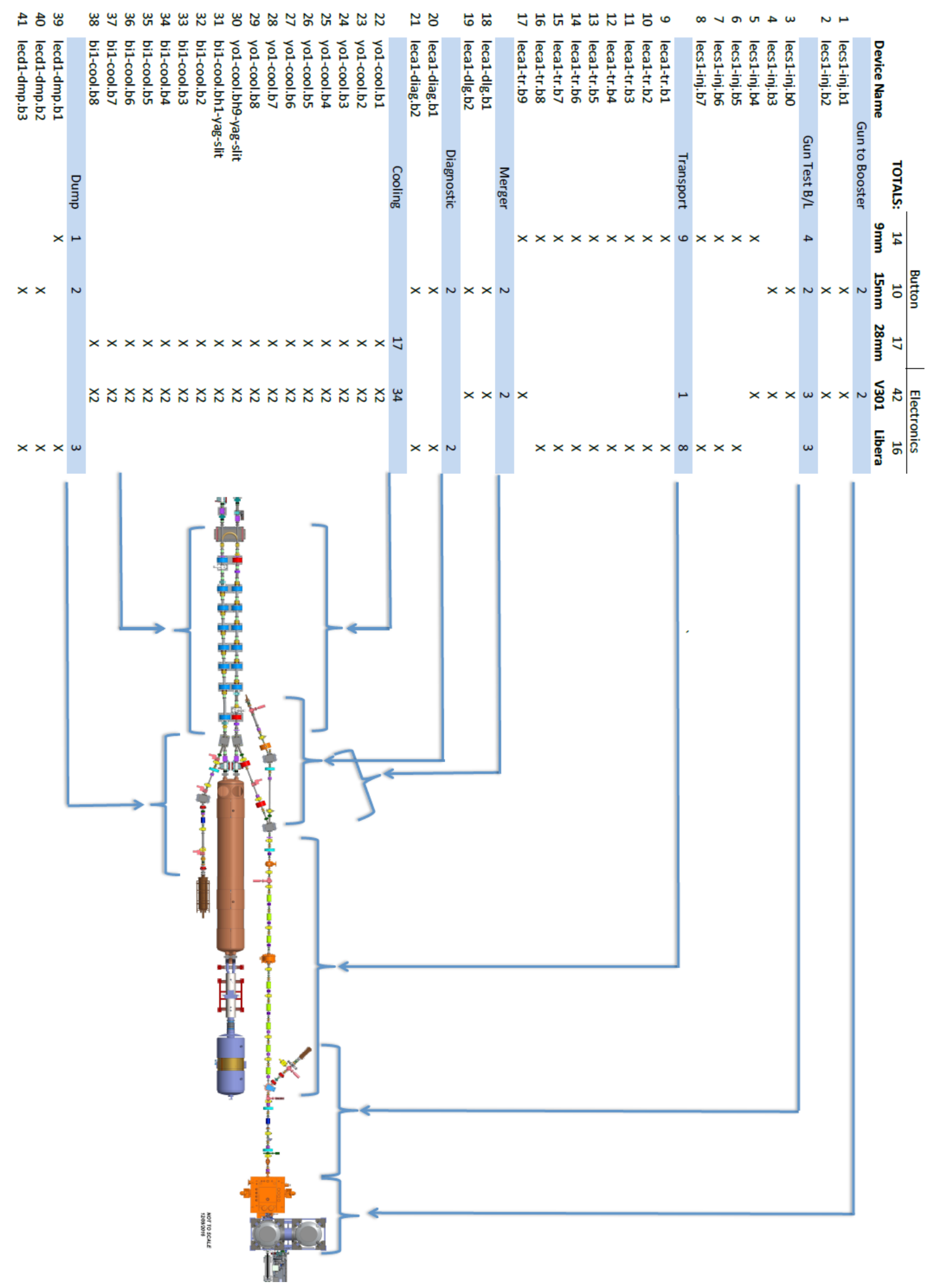

Figure 11: Layout of LEReC BPMs. 
The locations of LEReC magnets and the list of magnets that will be monitored by the MPS are shown in Fig. 12 and Table 5 respectively.

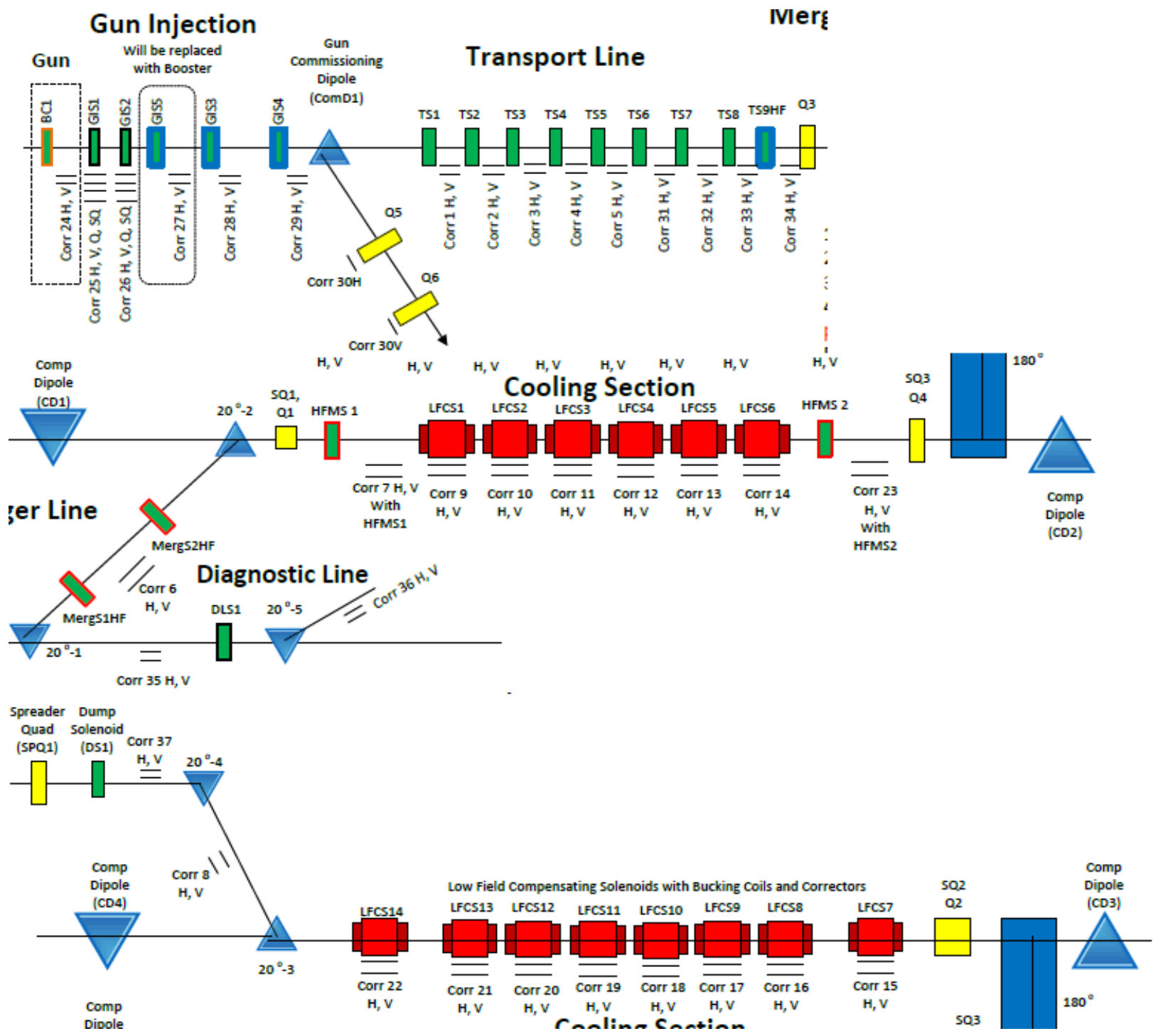

Figure 12: Layout of LEReC magnets. 
Needed for Gun Injection test Fall 2016

\begin{tabular}{|l|l|r|l|r|r|}
\hline Magnet Name & Type & Stability (ppm) & Model Number & Max Current of Current Sensor (A) & PS Max I (A) \\
\hline Corr 24H & LEM & 1000 & LTS 6-NP (LA 25-P) & 1 & 1 \\
\hline Corr 24V & LEM & 1000 & LTS 6-NP (LA 25-P) & 1 & 1 \\
\hline Corr25H & LEM & 1000 & LTS 6-NP (LA 25-P) & 1 & 1 \\
\hline Corr25V & LEM & 1000 & LTS 6-NP (LA 25-P) & 1 & 1 \\
\hline ComD1 & LEM & 1000 & LA 25NP & 25 & 25 \\
\hline
\end{tabular}

Note: LTS 6-NP is a voltage output LEM. It will eventually be replaced with an LA 25-P current output LEM

\section{Needed after Gun Injection test Fall 2016}

\begin{tabular}{|l|l|r|l|r|r|}
\hline Magnet Name & Type & Stability (ppm) & Model Number & Max Current of Current Sensor (A) & PS Max I (A) \\
\hline Corr26H & LEM & 1000 & LTS 6-NP (LA 25-P) & 1 & 1 \\
\hline Corr26V & LEM & 1000 & LTS 6-NP (LA 25-P) & 1 & 1 \\
\hline GIS1 & LEM & 1000 & LA 25NP & 12 & 12 \\
\hline GIS2 & LEM & 1000 & LA 25NP & 10 \\
\hline $20^{\circ}-1$ & LEM & 1000 & LA 25NP & 12 & 10 \\
\hline $20^{\circ}-2$ & LEM & 1000 & LA 25NP & 12 & 10 \\
\hline $20^{\circ}-3$ & LEM & 1000 & LA 25NP & 12 & 10 \\
\hline $20^{\circ}-4$ & LEM & 1000 & LA 25NP & 10 \\
\hline $20^{\circ}-5$ & LEM & 1000 & LA 25NP & 12 & 12 \\
\hline 180 & DCCT & $<100$ & IT 60-S & 10 & 10 \\
\hline
\end{tabular}

Note: LTS 6-NP is a voltage output LEM. It will eventually be replaced with an LA 25-P current output LEM

Table 5: Magnets monitored by the MPS.

The location of the vacuum gauges for 2017 gun test run is shown in Fig. 13.

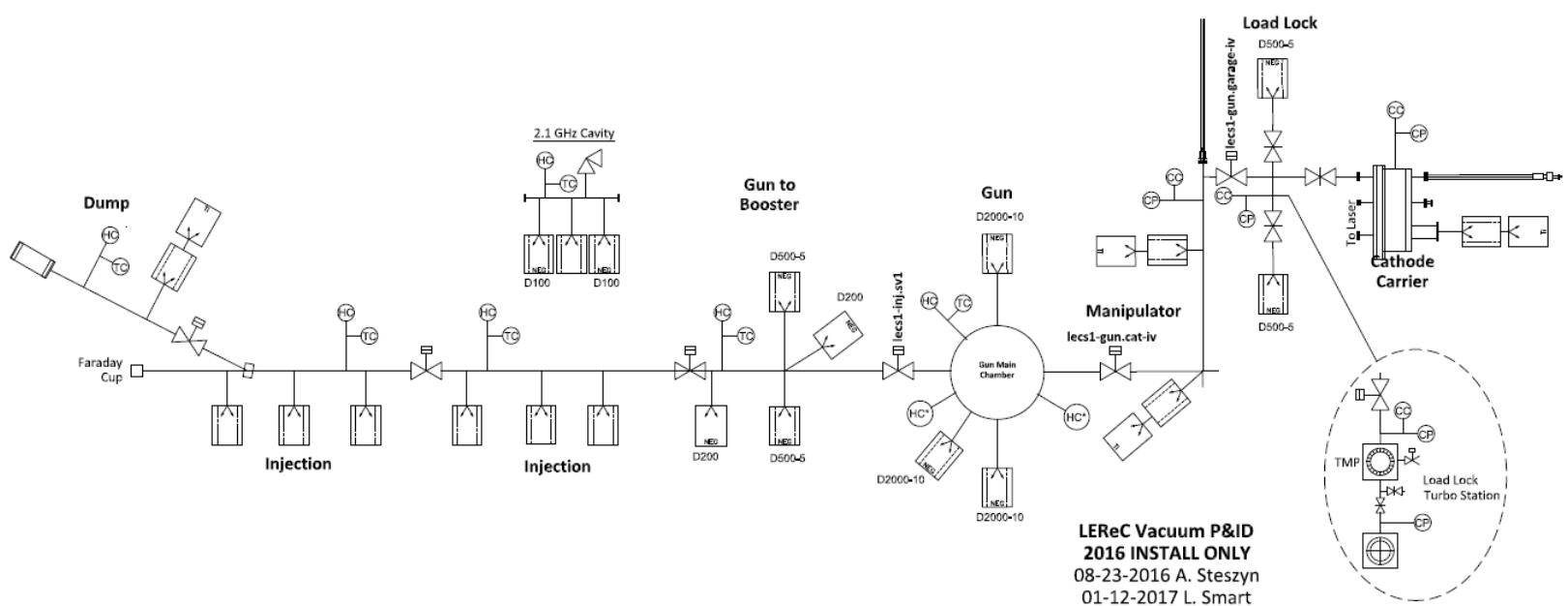

Figure 13: Approximate locations of the vacuum gauges for the gun test beam line. 
The MPS will rely on four FCTs one located at the beginning of the accelerator (see Section 3.1 for details) and three other located at three dumps. The first FCT is the critically important device that is used to measure beam current (see Section 4.2 for details) and to determine what beam and equipment manipulations are allowed at the moment.

The other FCTs are used in combination with the first one for differential measurements and determination of the beam losses. While the FCT differential measurement will be a subject of experimental studies it is important to point out that both the longitudinal shape and the length of the individual bunch significantly changes throughout the LEReC. For instance, Fig. 14 shows how the longitudinal profile of the bunch changes from gun exit to the $180^{\circ}$ bend. Figure 15 shows bunch lengthening for various beam energies and bunch charges. This topic is discussed more in the next section.

(a)

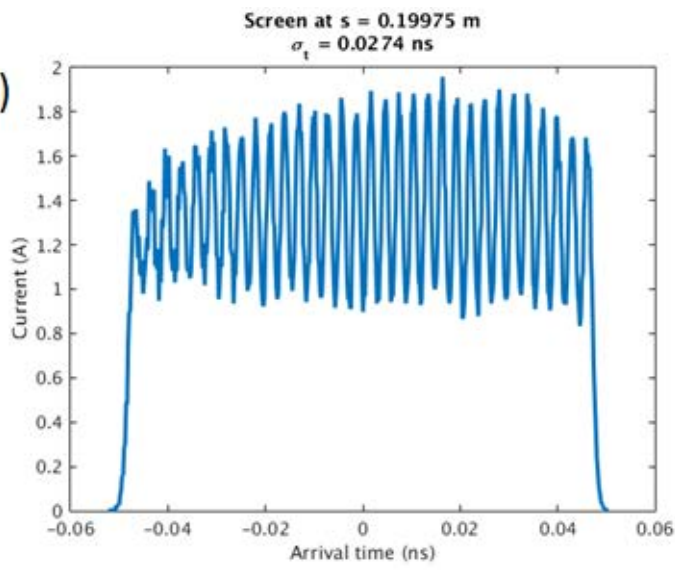

(c)

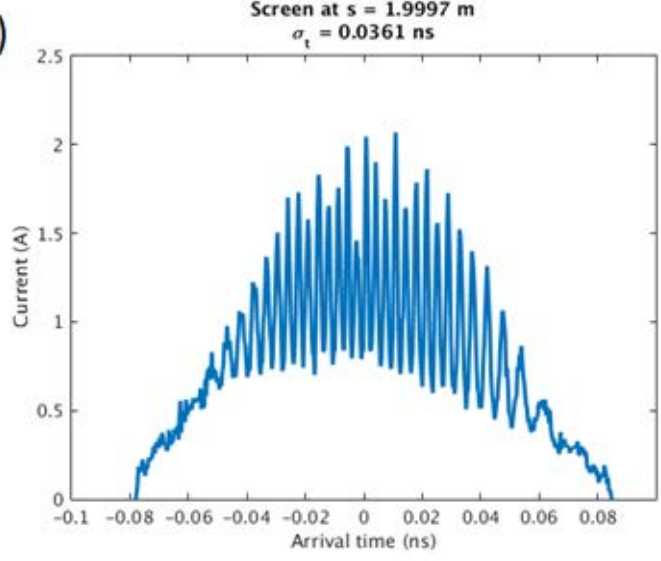

(b)

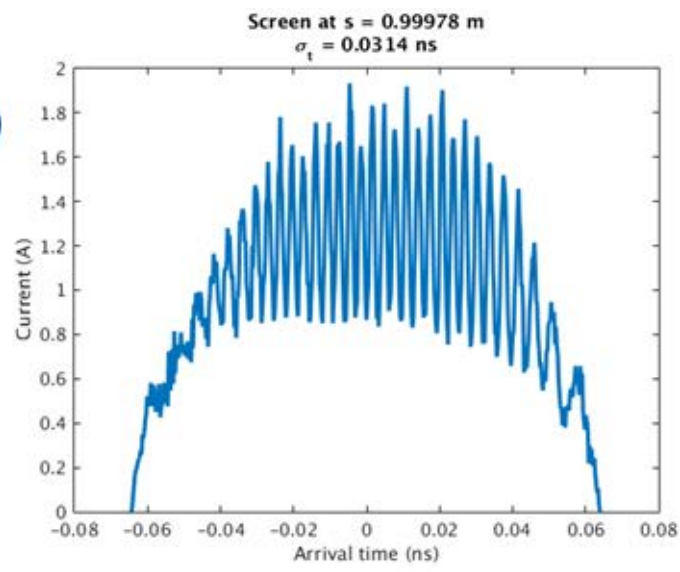

(d)

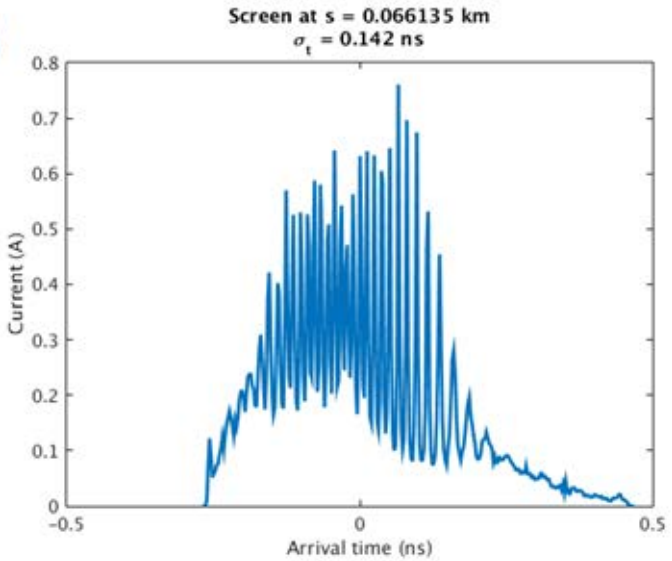

Figure 14: 1.6 MeV 100 pC bunch longitudinal profile at gun exit (a), SRF Booster entrance (b), Booster exit (c) and $180^{\circ}$ bend (d). 


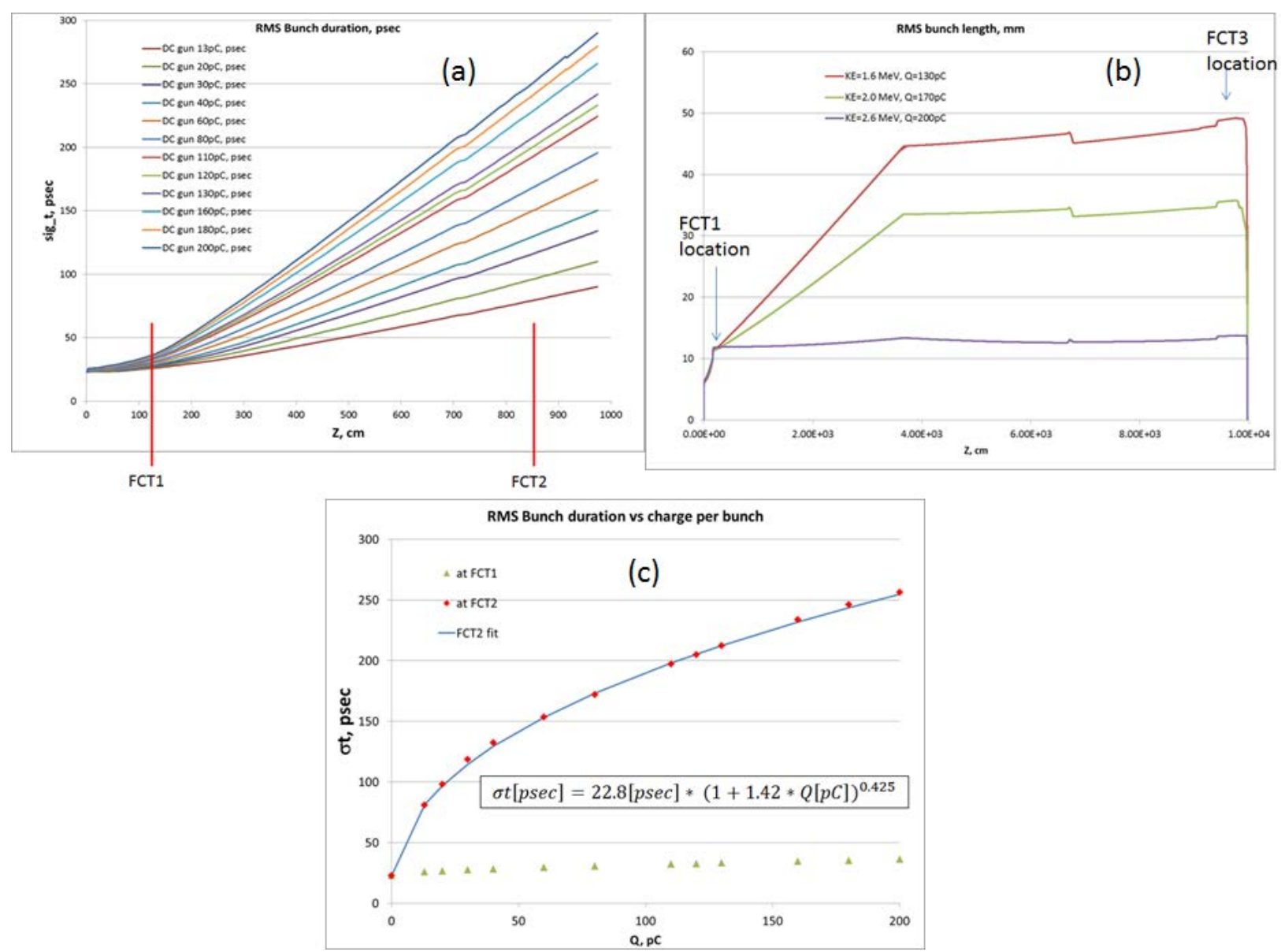

Figure 15: Bunch length for various charges in gun test setup (a) and $200 \mathrm{pC}$ charge and various energies in the full LEReC (b). Plot (c) shows bunch length vs. bunch charge dependence derived from (a).

\subsection{LEReC FCT}

The output of the LEReC FCT is a $704 \mathrm{MHz}$ wave with amplitude proportional to the bunch charge. The signal is sent to the peak detector which converts it to DC signal representing the instantaneous charge of the beam passing the FCT. The DC signal is digitized and used to determine the current level, which is supplied to the MPS controller.

While the FCT essentially measures the instantaneous beam current we need to have the instantaneous measurement of an average beam current. In other words, ideally we want to know that the average current level has changed at the moment it has changed as Fig. 16 demonstrates. 


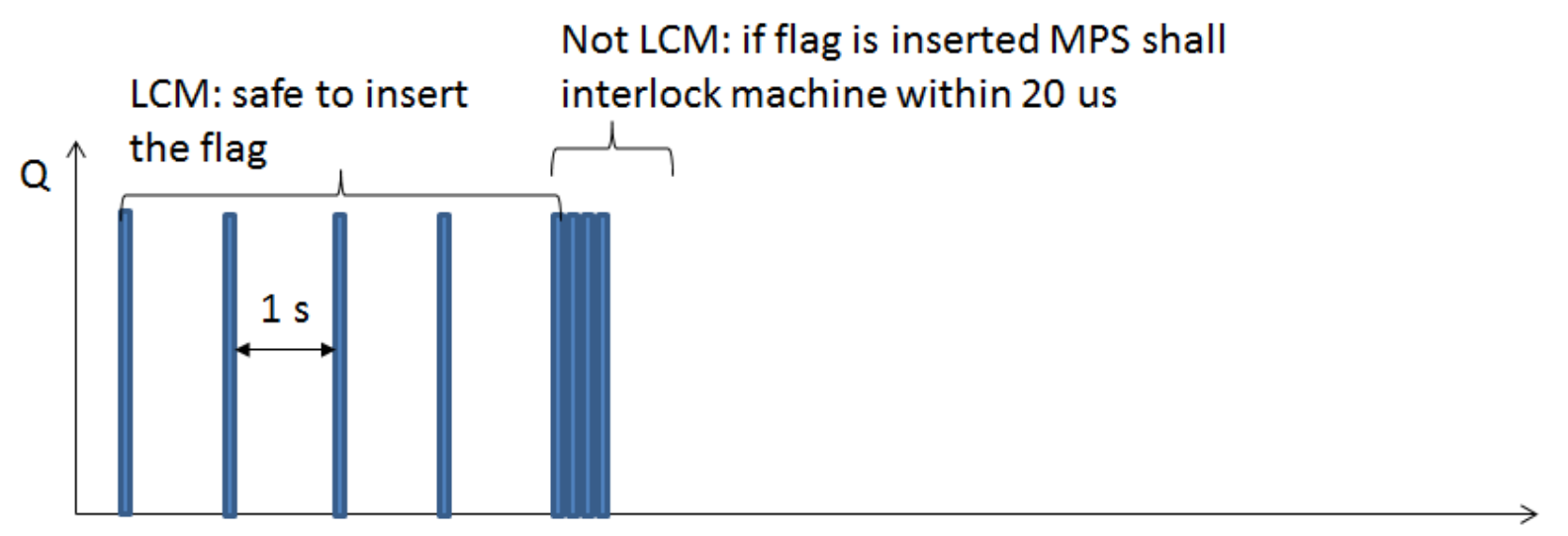

$\mathrm{t}$

Figure 16: MPS needs to know that the average beam current has changed within a few microseconds.

To achieve this goal we suggest measuring the total charge accumulated in the moving window of some length T (Fig. 17). This charge will be defining our current levels for the MPS. It is worth noticing that the window of length $T$ overestimates average current for repetition rate $<1 / T$. We suggest to have $\mathrm{T}=5 \mathrm{~s}$, which is the longest expected period in all of our beam modes (see Section 1.2 for details).

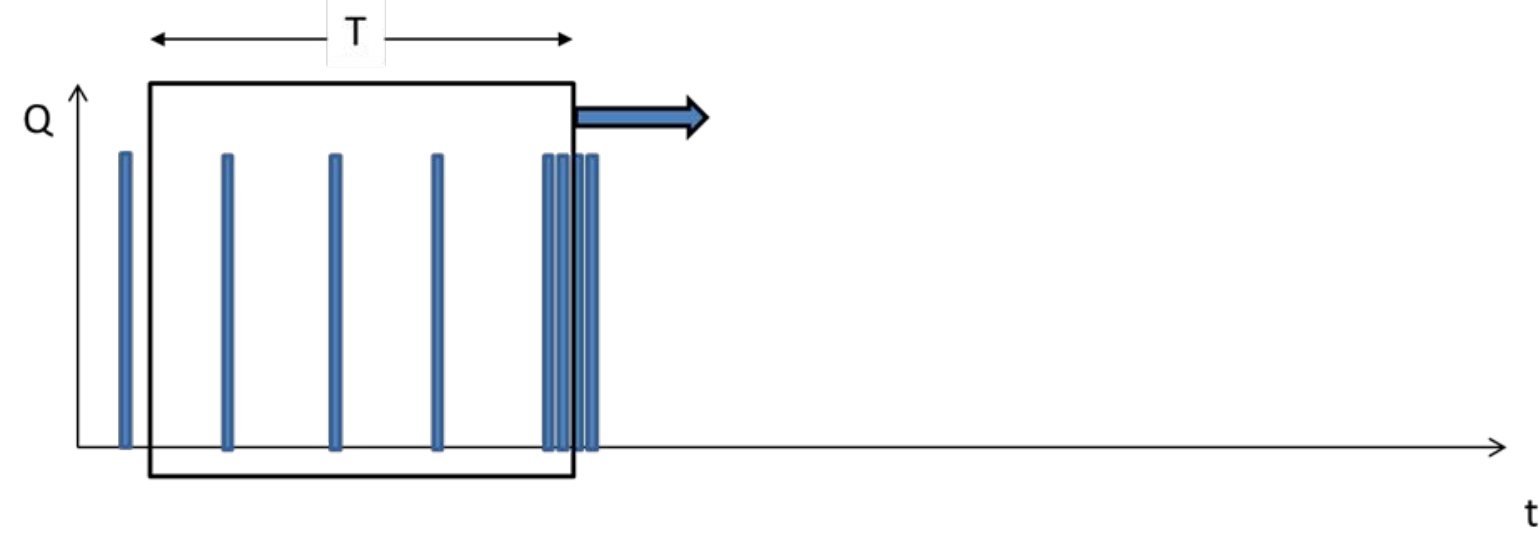

Figure 17: The concept of the FCT measurement for the LEReC MPS.

The equivalent charges corresponding to various current levels discussed throughout this paper are listed in Table 6. 


\begin{tabular}{|c|c|c|c|c|}
\hline Beam mode & Machine mode & current & $\begin{array}{l}\text { Q accumulated in } \\
5 \text { s long window }\end{array}$ & Comments \\
\hline LCM & Flags, valves, etc. & $6 \mathrm{nA}$ & $30 \mathrm{nC}$ & \\
\hline \multirow[t]{2}{*}{ USOM } & Flags, valves, etc. & $40 \mathrm{nA}$ & $200 \mathrm{nC}$ & \\
\hline & & $1 \mathrm{uA}$ & $5 \mathrm{uC}$ & Initial I Ioss \\
\hline \multirow[t]{4}{*}{ RFSM } & RF line flag/dump & 3 uA & $15 \mathrm{uC}$ & \\
\hline & & 80 uA & $400 \mathrm{uC}$ & FCT-PD threshold \\
\hline & & $600 \mathrm{uA}$ & $3 \mathrm{mc}$ & $\begin{array}{l}I_{\max } \text { - threshold for studies } \\
\text { of tolerable } I_{\text {loss }} \text { and BLM } \\
\text { settings }\end{array}$ \\
\hline & $10 \mathrm{~kW}$ dump & $4 \mathrm{~mA}$ & $20 \mathrm{mc}$ & $\begin{array}{l}\text { current/charge are } \\
\text { calculated for } 2.6 \mathrm{MeV} \text { beam }\end{array}$ \\
\hline $\mathrm{TM}, \mathrm{HCM}$ & $140 \mathrm{~kW}$ dump & $55 \mathrm{~mA}$ & $275 \mathrm{mc}$ & \\
\hline CWM & $140 \mathrm{~kW}$ dump & $85 \mathrm{~mA}$ & $425 \mathrm{mc}$ & \\
\hline
\end{tabular}

Table 6: Charge in $5 \mathrm{~s}$ window defines the MPS current levels. Please notice that not all of the listed charges shall be supplied to MPS controller, some of them are presented here just for reference.

The bench test of the FCT was performed with $704 \mathrm{MHz}$ Gaussian-like bunches (FWHM 500 ps) produced by the generator. The bunches were either stacked into the "macrobunches" of arbitrary length or produced as CW (either $704 \mathrm{MHz}$ CW or continuous $9 \mathrm{MHz}$ train of 42 ns long $704 \mathrm{MHz}$ macrobunches). Such setup was feasible for the equivalent charge per bunch in the range of $1 \mathrm{pC}-20$ pC. To emulate the higher charges the pure sinusoidal $704 \mathrm{MHz}$ wave was used.

The rise time of the DC signal out of converter was $\sim 300 \mathrm{~ns}$, which is equivalent to 3 macrobunches of LEReC beam. For 42 ns long train of pulses (single LEReC macrobunch) the accuracy of the measurement, which is defined by the signal to noise ratio, was $\sim 1 \mathrm{pC} /$ bunch. Apparently, as the length of the pulse train was increased the accuracy was getting better.

For the MPS purposes it is fair to declare that the accuracy of the FCT measurements is $1 \mathrm{pC} /$ bunch.

The results of the bench test are presented in Fig. 18 (a).

Figure 18 (b) shows the response of the FCT to bunches, which length increases with the bunch charge. To produce the measurement presented in Fig. 18 (b) the pulses emulating the bunches were elongated with the increase of pulse amplitude in accordance to dependence illustrated in Fig. 15. 


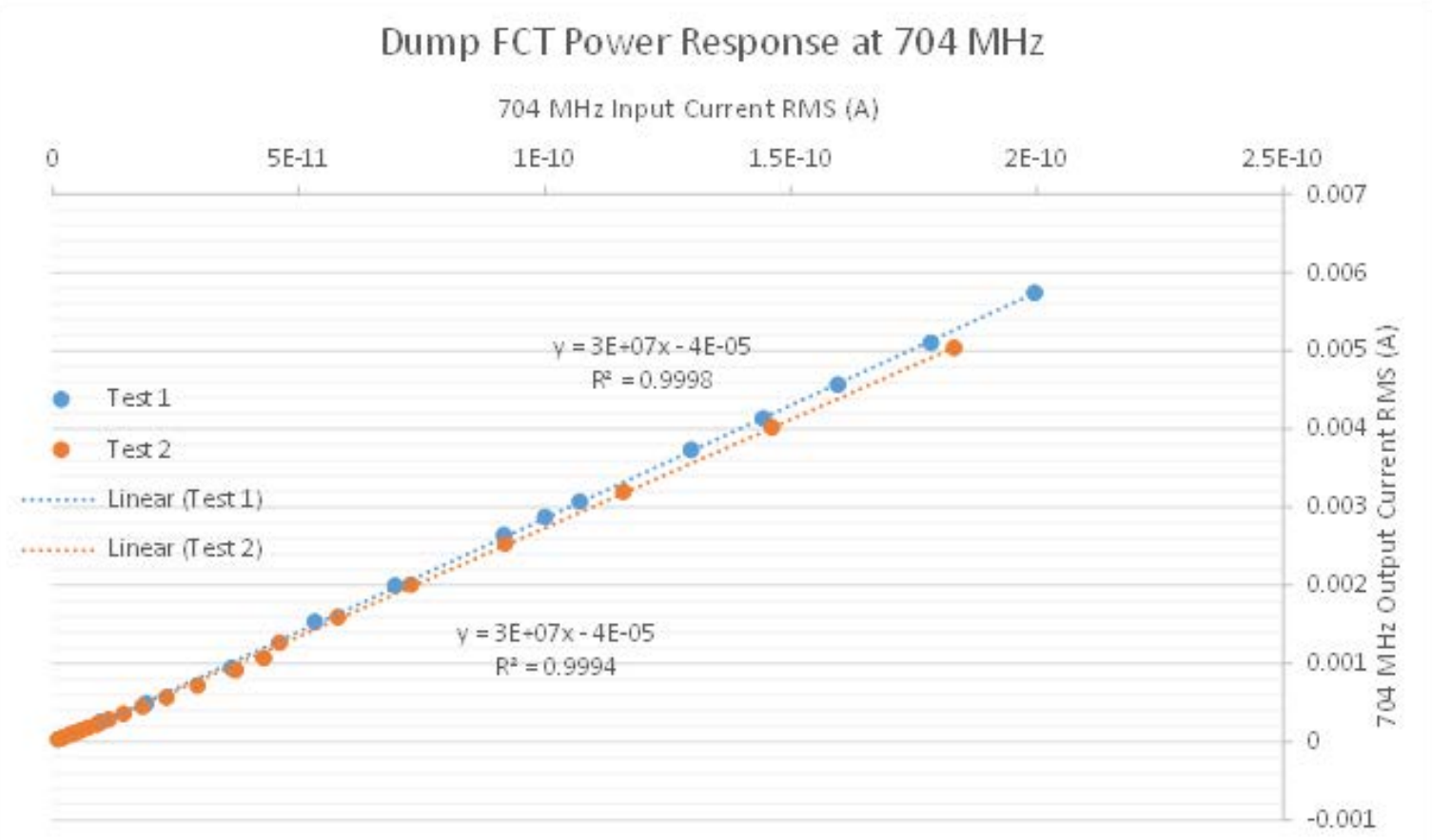

(a)

(b)

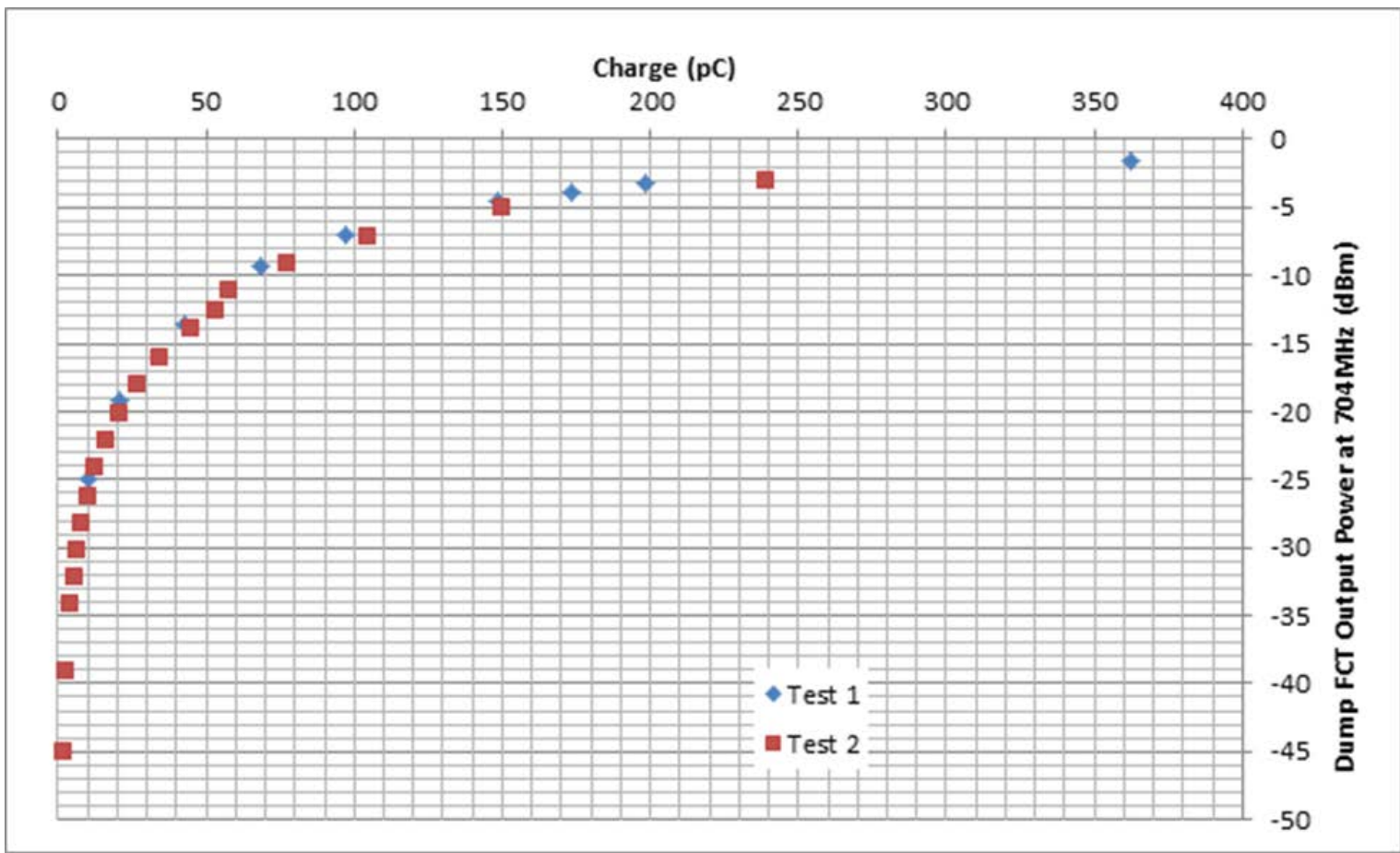

Figure 18: (a) FCT response to various bunch charges. (b) FCT response to various bunch charges with bunch length varying in accordance to plot $15 \mathrm{c}$.

\section{Laser - MPS interface}

The schematic of the laser is shown in Fig. 19. 


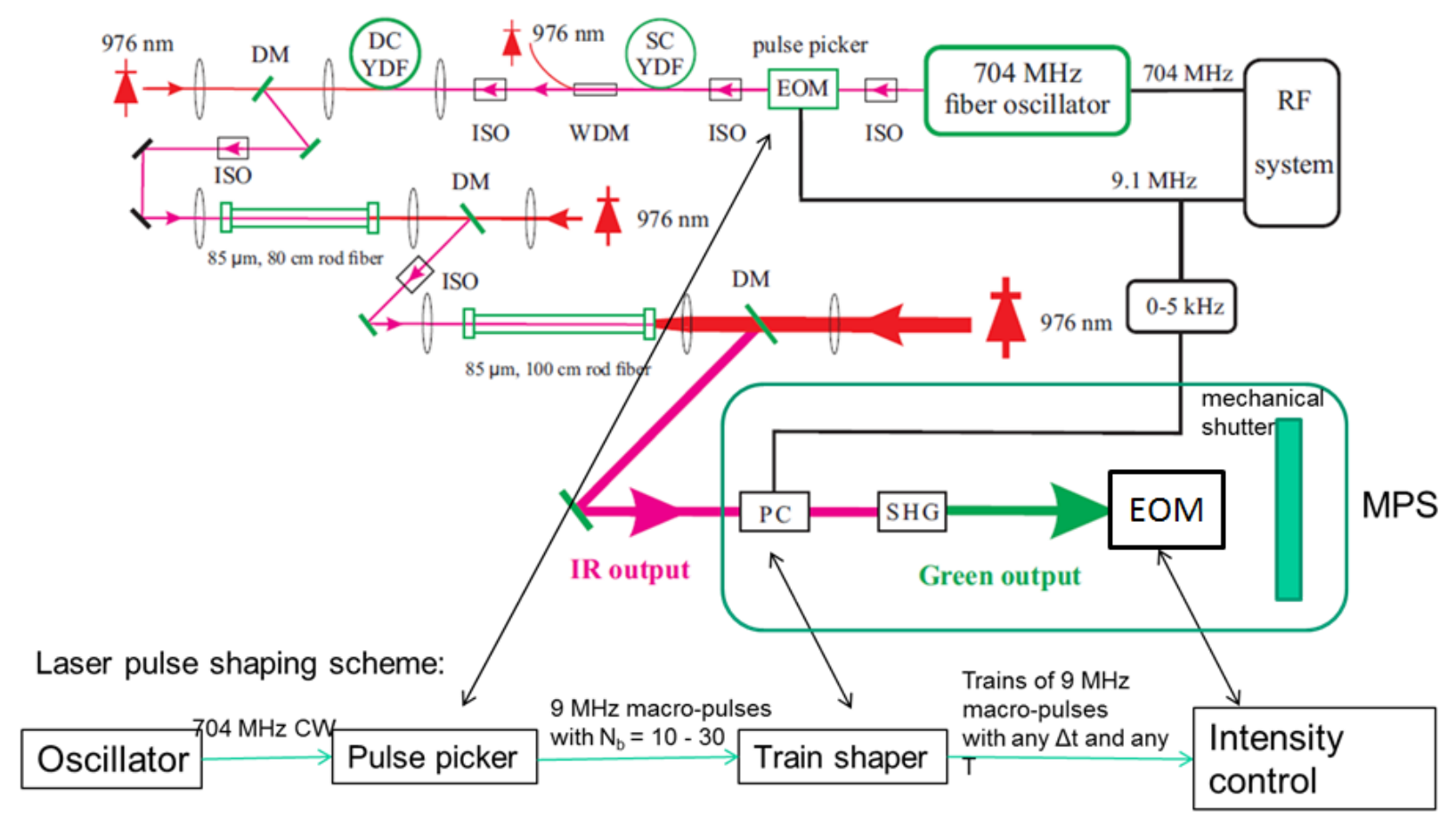

Figure 19: LEReC laser setup.

The CW 704 laser pulses are first sent to the pulse picker. The pulse picker is an Electro-Optic Modulator (EOM) with the fast ( $1 \mathrm{~ns})$ rise/fall time. It has to be fine-tuned for high extinguish ratio. Therefore, one cannot simply switch the pulse picker between the $704 \mathrm{MHz} C W$ and back to $9 \mathrm{MHz}$ macro-bunches. Instead, one must physically bypass the EOM to switch to the CW mode.

After the pulse picker the train of $9 \mathrm{MHz}$ macrobunches (or true $\mathrm{CW}$ if the pulse picker is bypassed) is amplified and delivered to the train shaper. The train shaper is a Pockels cell (PC) followed by the halfwave plate (HWP). Depending on the HWP angle the PC either passes the macro-bunches through when the voltage is applied (this mode is used to create the trains of macro-bunches of particular length with some repetition rate) or blocks the macro-bunches when the voltage is applied (this mode is used to create a continuous train of macro-bunches with short gaps for ion clearing).

The PC can withstand the high voltage only for $5 \%$ of its switching period. That is, if we want to have trains with $1 \mathrm{~Hz}$ repetition rate then the length of the trains cannot exceed $50 \mathrm{~ms}$. This is exactly the operation setup that will be used for the first few months of the gun test run.

Intensity Controller consists of another EOM for intensity stabilization and a HWP for intensity limitation. The EOM is used to cut a few percent of laser intensity to smooth the intensity variation. The remotely controlled HWP polarizer is used to set required laser intensity.

Finally, the laser can be interrupted by the slow (as compared to the fast shutter used only to increase the pulse extinguish ratio) mechanical shutter installed downstream of the intensity control system.

The MPS is interlocking the machine by closing the train shaper PC, closing the EOM and closing the mechanical shutter. The schematic of the laser-MPS interface is shown in Fig. 20. 


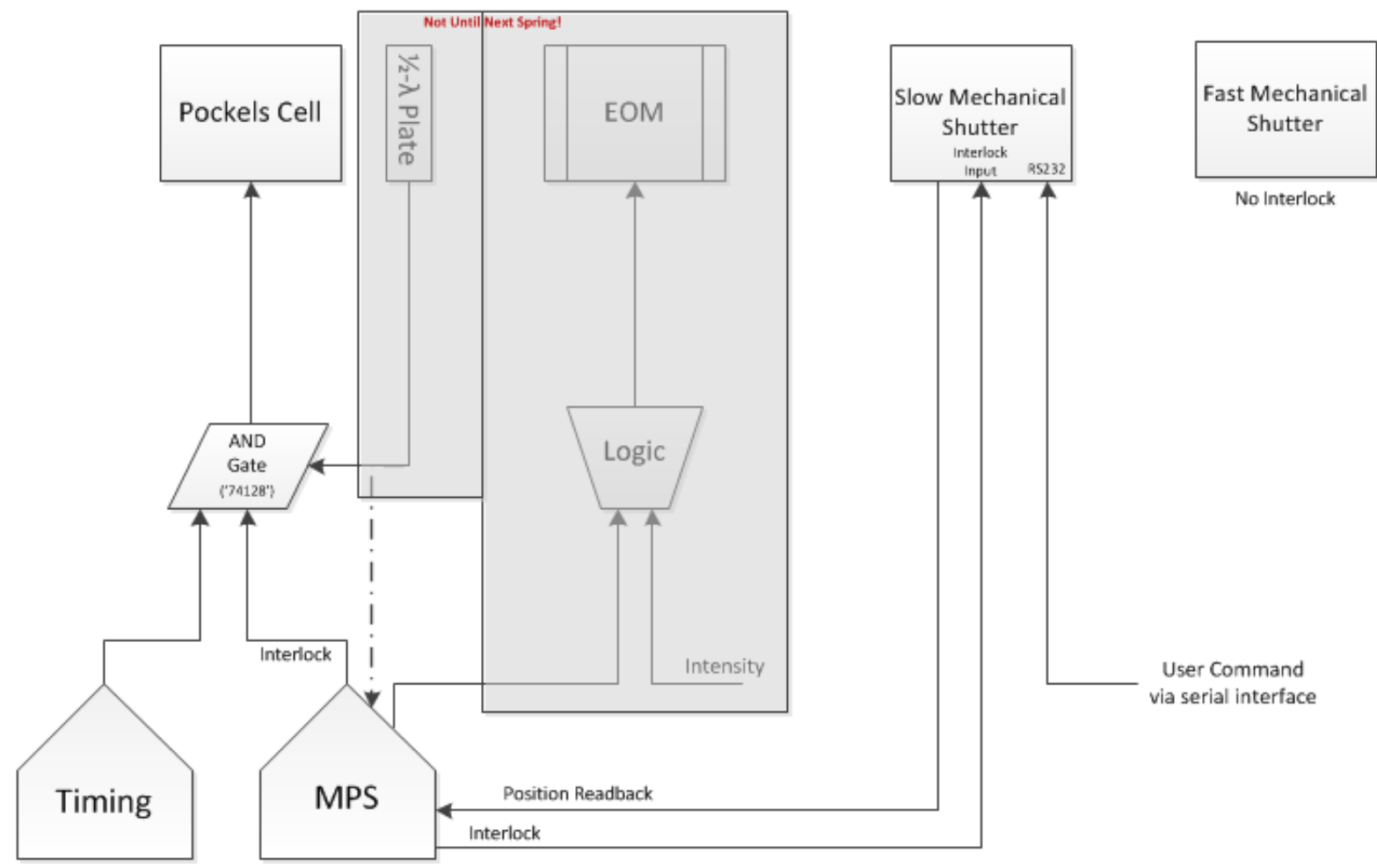

Figure 20: LEReC laser-MPS interface.

\section{Gun-MPS interface}

The LEReC gun has a standalone protection system. Yet, the MPS under consideration still interacts with the gun.

First of all the MPS receives the status of the gun high voltage power supply (HVPS). The MPS must know whether the HVPS is enabled or disabled and whether its readback is 0 (for disabled HVPS) or it is at the nominal value (for enabled HVPS).

Another task of the MPS is to send interlock signal to the HVPS when the gun vacuum gauge reads the value above the allowed threshold.

The MPS interlocks the laser during a trip of the HVPS in order to avoid making beam of wrong energies during the decay of the cathode voltage due to a trip of the HVPS. Once the HVPS is at OV, there is no longer a reason to disable beam and the machine immediately is considered to be in Laser Alignment Mode (since there is no more accelerating voltage).

The logic of the gun-MPS interaction will be discussed in more details in Section 7. 


\section{MPS logic}

\subsection{MPS schematic}

The overall MPS schematic summarizing the discussed material up to this point is presented in Fig. 21.

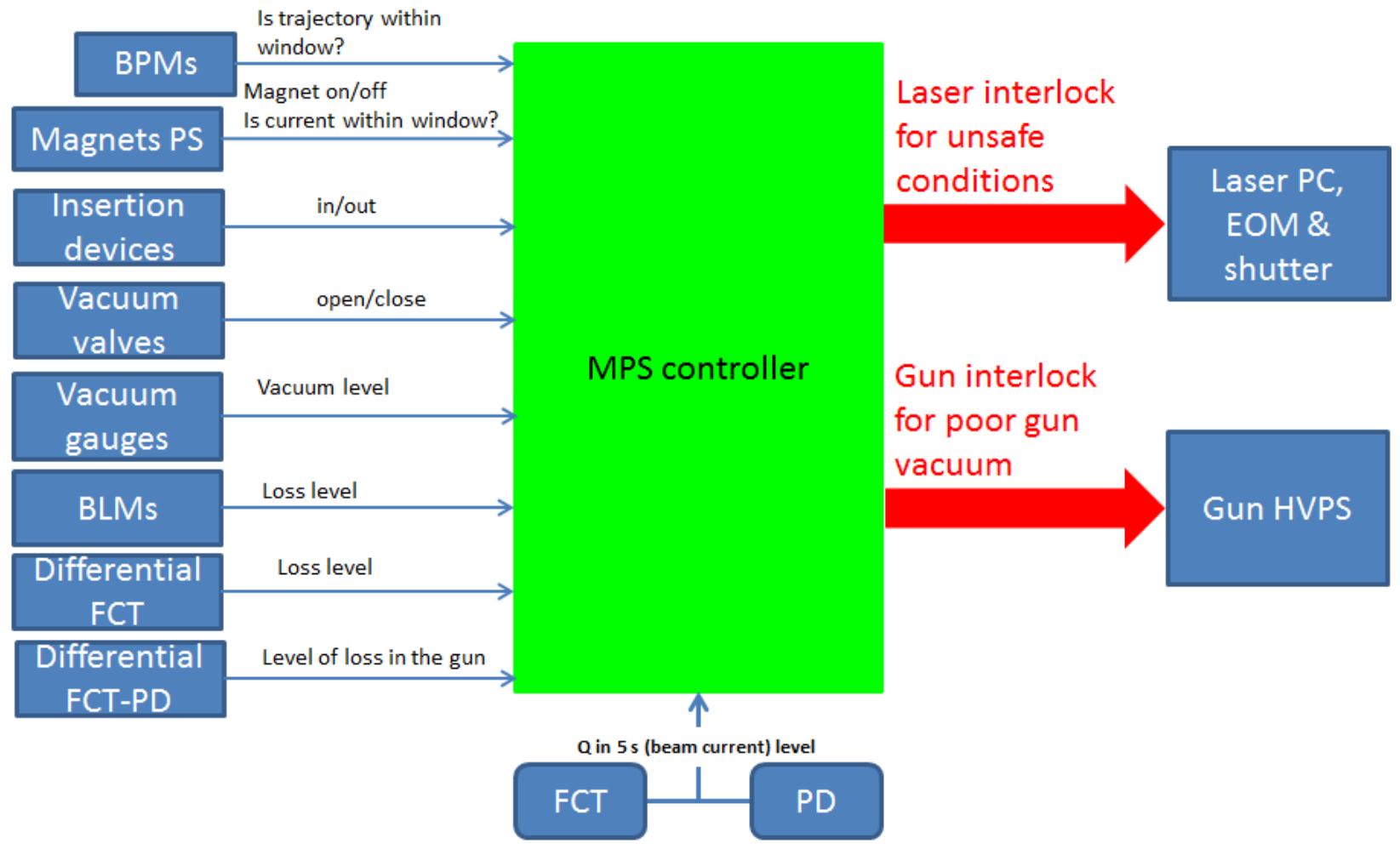

Figure 21: Schematic of LEReC MPS

Below we describe the logic of the MPS controller.

\subsection{Concept of MPS logic}

The MPS assesses the surface, which the beam is hitting, from the settings of the dipoles and from what insertion devices are inserted into the beamline. These inputs to the MPS are called "qualifiers" and the surface hit by the beam defines the "machine mode" (MM).

The operation in each particular MM is allowed below certain current level only.

The actual beam current ( $Q$ in $5 s$ ) is calculated from the FCT and the PD readings.

The MPS compares the measured beam current to the allowed current level and if the measured current exceeds the limit set for the present MM then the MPS trips the beam. Another cause for the MPS to trip the machine above certain current level is the BPM readings or magnet PS readings outside of the allowed range.

Finally, above certain current level the MPS trips the beam if the loss either from the BLMs or differential FCT or FCT-PD signals is above the $I_{\text {loss }}$. 
The last but not the least the MPS allows two additional modes of operation: the "isolation mode" and "laser alignment mode".

In the isolation mode the laser shutter is closed so that the gun and the laser conditioning can be performed independently. The qualifier for this mode is the status of the laser shutter.

In the laser alignment mode the gun high voltage (HV) is turned off, so that the laser can be aligned on the cathode. The status of the gun HV is the qualifier for this mode.

The concept of the MPS logic can probably be explained the best by an example illustrated by Fig. 22.

Assume that an operator works in RFSM.

If gun diagnostic bend and first merger bend are "off" while RF diagnostic bend is "on" and RF YAG is inserted then MPS decides that it is working in respective MM.

The $Q$ in $5 \mathrm{~s}$ (Q5) for this MM is $15 \mathrm{uC}$. If operator runs (with $0.2 \mathrm{~Hz}$ ) the beam with $200 \mathrm{pC} / \mathrm{bunch}$ and at some point exceeds the allowed length of the macro-bunch train ( 250 us) by 50 us then at that instant the measured Q5 becomes larger than $15 \mathrm{uC}$ and MPS drops the beam by closing the laser shutter.

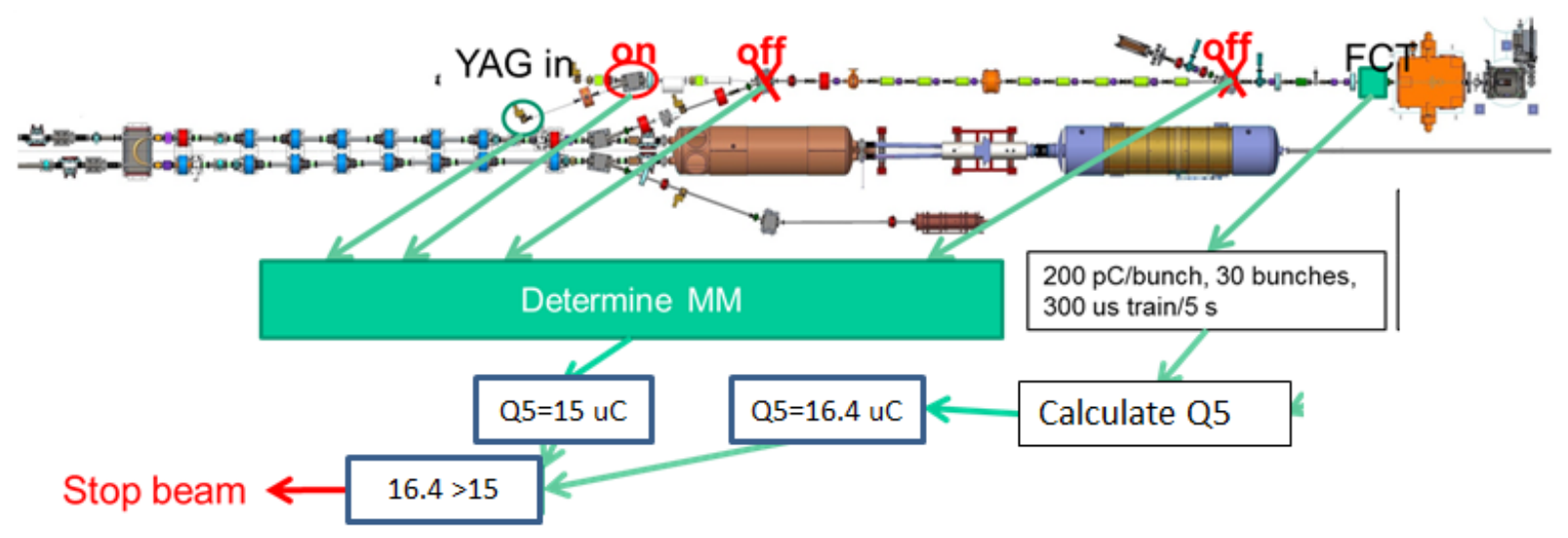

Figure 22: Example of MPS logic.

\subsection{MPS Logic}

In this section we consider the logic for the 2017 gun test run. It is a scaled down logic of the full LEReC MPS but it has all the bells and whistles of the full system.

Figure 23 and Table 7 show the block diagram of the complete MPS for the gun test run. 


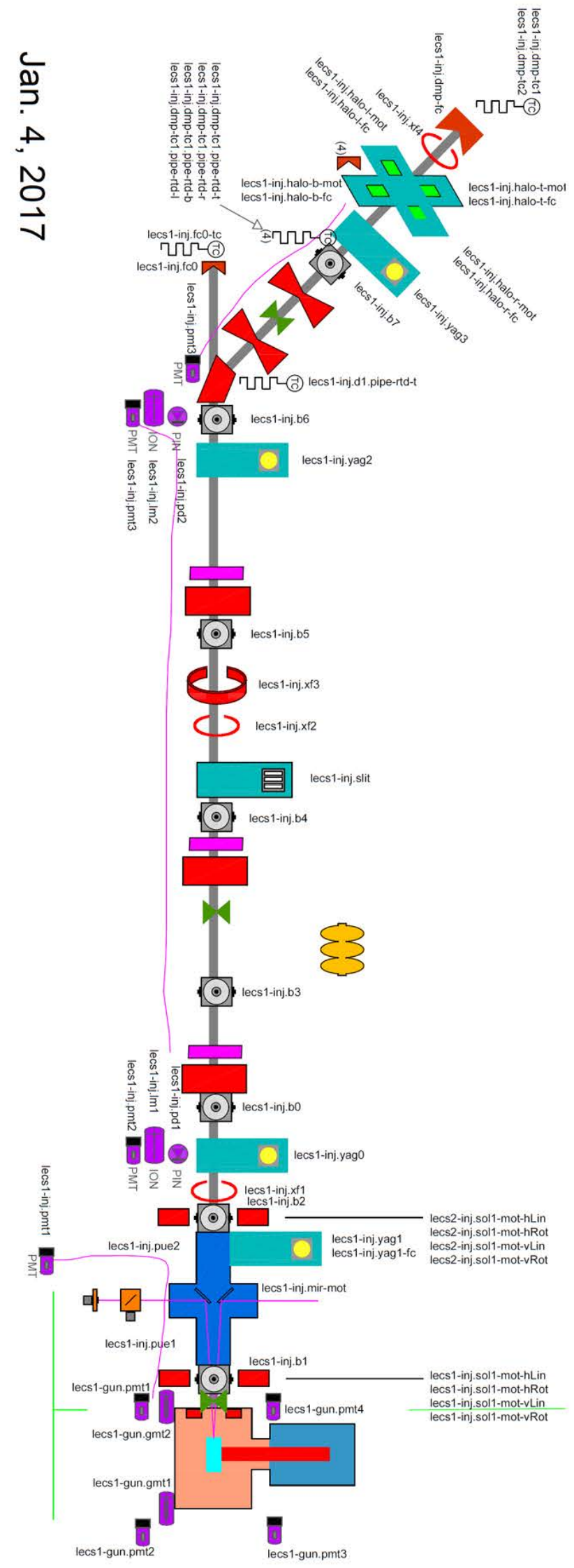




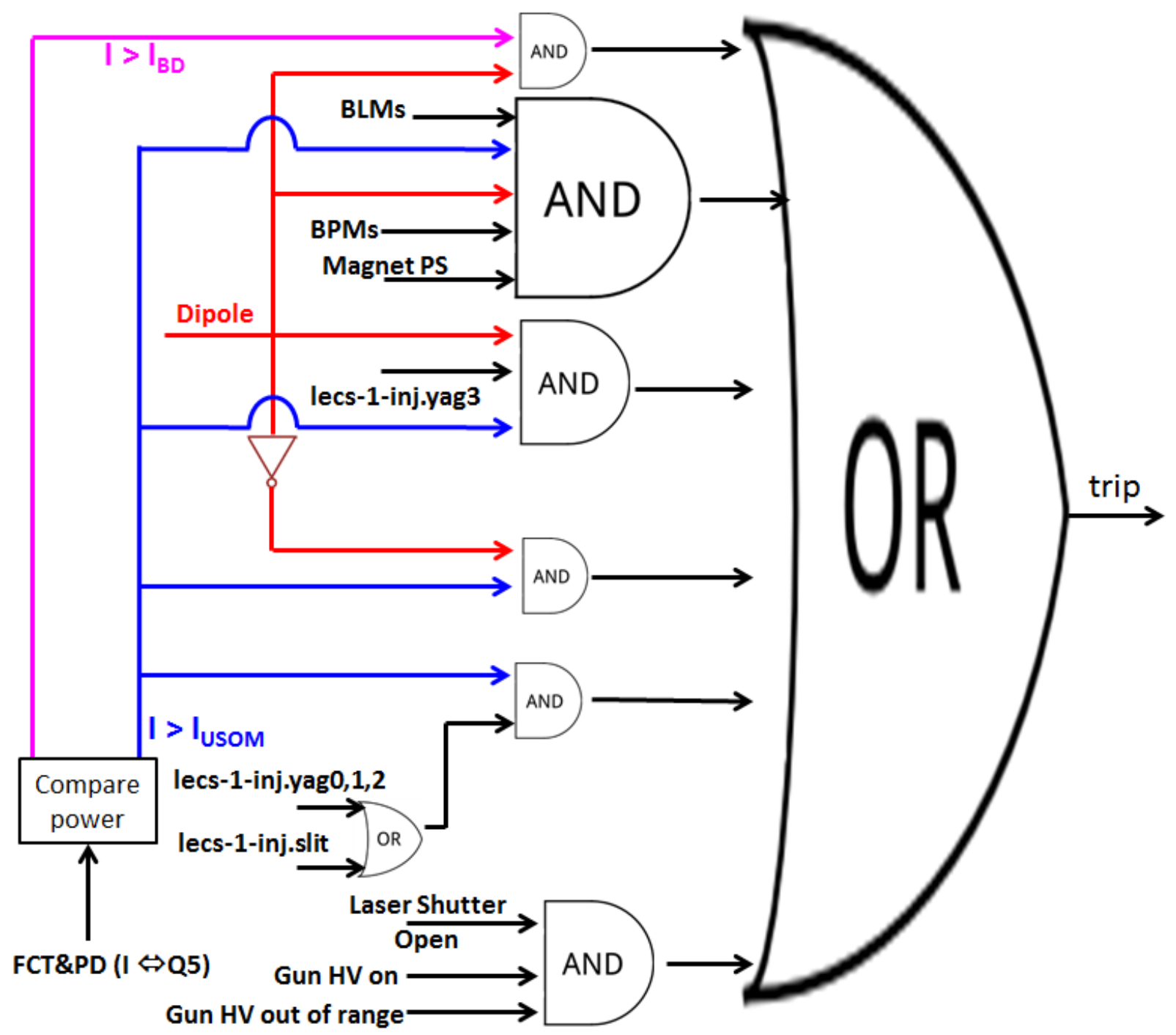

Figure 23: Block diagram of the MPS logic for the gun test run. 


\section{0}

\begin{tabular}{|l|l|l|}
\hline Dipole & Off & On \\
\hline BPMs & In range & Not in range \\
\hline Magnet PS & In range & Not in range \\
\hline lecs-1-inj.yag3 & Out & In \\
\hline lecs-1-inj.yag0,1,2 & Out & In \\
\hline lecs-1-inj.slit & Out & In \\
\hline BLMs & $\begin{array}{l}\text { Losses are } \\
\text { below limit }\end{array}$ & $\begin{array}{l}\text { Losses are } \\
\text { above limit }\end{array}$ \\
\hline
\end{tabular}

Table 7: The states of the MPS inputs on Fig. 23.

The logic flowchart of the MPS for the gun test run is shown in Fig. 24-26. Figure 24 shows FCT and PD inputs to the MPS. Figure 25 shows the logic that determines the laser alignment and isolation modes. Figure 26 shows the MPS logic for beam operation. 
* These inputs are not maskable.

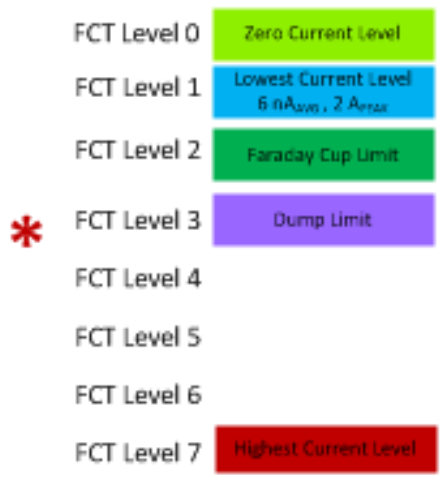

MPS will identify the one FCT level that is active and declare this to be the allowable beam current level. If there is no active level or there is more than one active level, MPS will interlock the laser and will not release it until only FCT Level 0 becomes active.

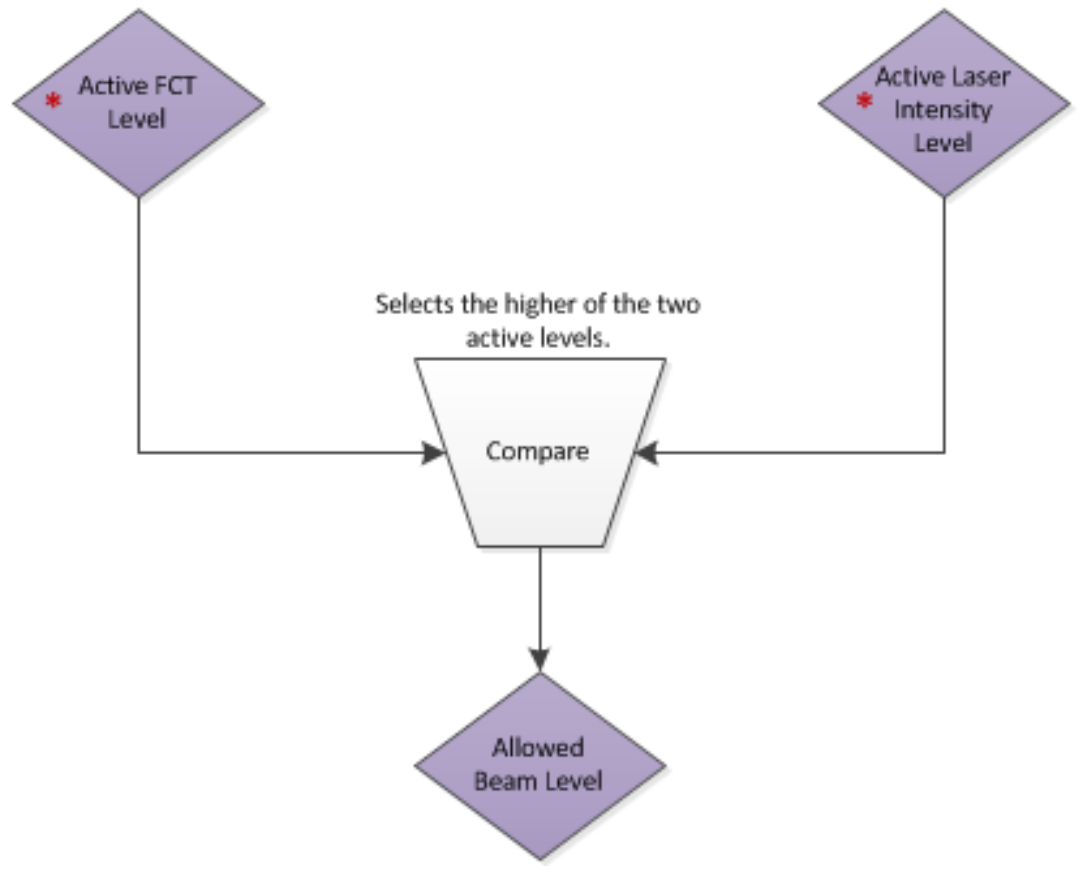

Figure 24: The FCT and PD input to the MPS.

\section{* These inputs are not maskable.}

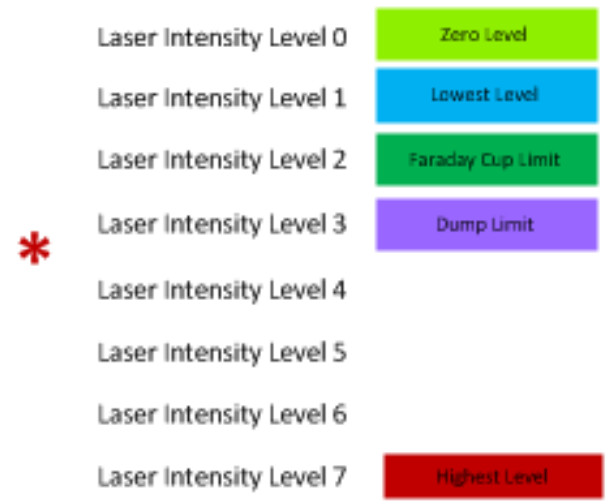

MPS will identify the one Laser Intensity level that is active and declare this to be the allowable laser power level. If there is no active level or there is more than one active level, MPS will interlock the laser and will not release it until only Laser Intensity Level 0 becomes active. 


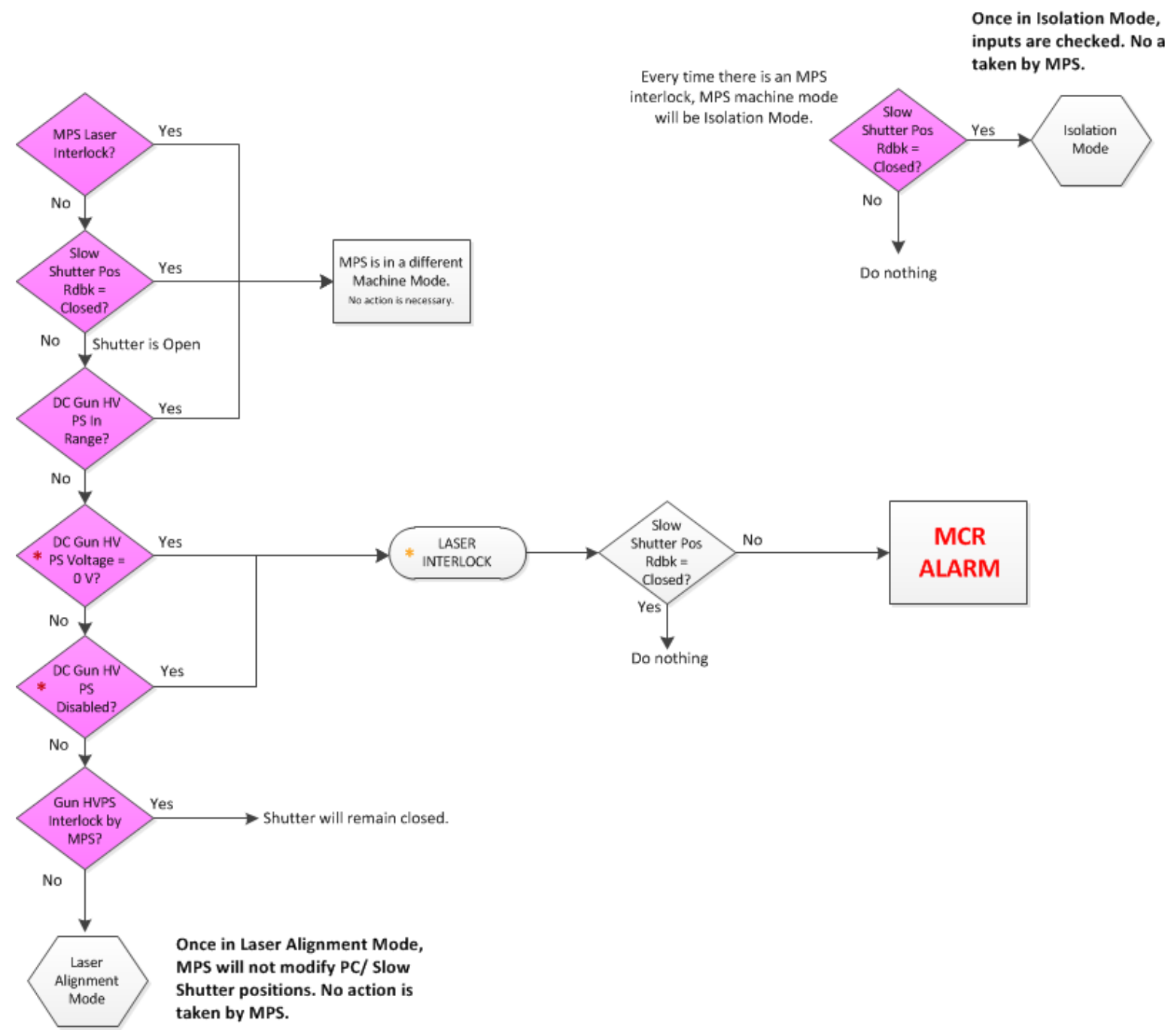

Figure 25: The MPS logic for laser alignment and isolation modes. 


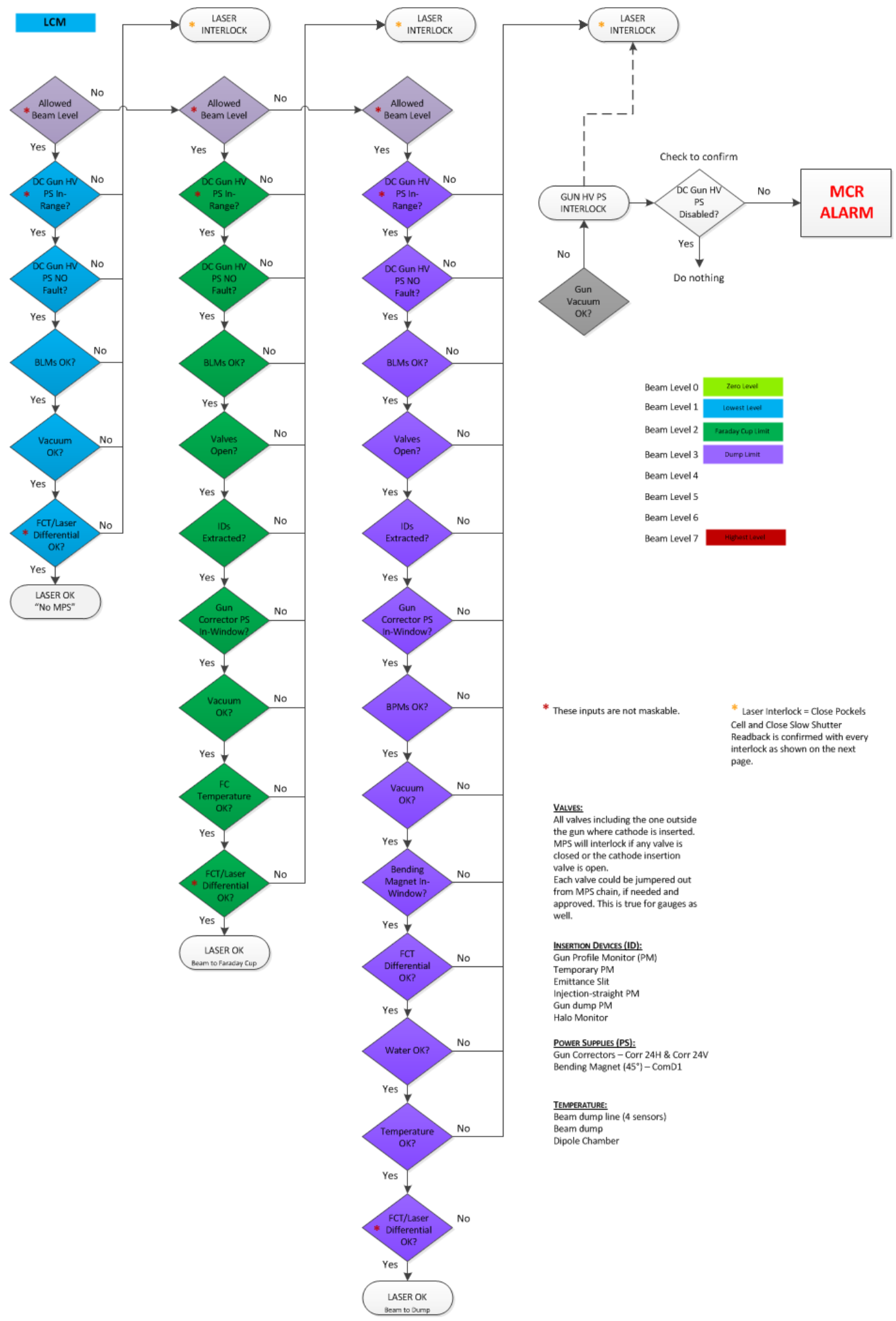

Figure 26: The MPS logic for beam operation. 


\section{Commissioning procedures}

\subsection{Integrated system test without beam (for 2017 run)}

\subsection{Purpose}

1.1.1 The purpose of this procedure is to check the integrity of the LEReC machine protection system (MPS). The steps outlined in this procedure aim to test two aspects of MPS functionality: The first is the interaction between the MPS controller, the MPS diagnostic subsystems, the laser, and the gun high-voltage power supply (HVPS). The second is to verify the logic of the MPS controller.

\subsubsection{Abbreviations and Symbols:}

$\mathrm{BD}-10 \mathrm{~kW}$ beam dump

BLM - beam loss monitor

BPM - beam position monitor

EOM - electro-optical modulator

FC - Faraday cup

FCT - fast current transformer

HVPS - high-voltage power supply

MPS - machine protection system

PC - laser Pockels Cell

PD - laser photodiode installed on the laser table in the tunnel

PS - power supply

Q5 - beam charge accumulated in $5 \mathrm{~s}$ window (the measure of the beam current)

IBD - allowed current threshold for the beam delivered to the beam dump

IFC - allowed current threshold for the beam delivered to the Faraday cup

lloss - threshold of the tolerable routine losses

Imax - threshold for studies of tolerable lloss and BLM settings

IUSOM - current threshold for the ultimately safe operation mode

\subsection{Responsibilities}

1.2.1 The LEReC Liaison Physicist is responsible for ensuring that the integrated system test is completed and that the MPS system is correctly configured. Problems with components of the system or issues that arise during testing should be reported to the Liaison Physicist as soon as possible to ensure that these issues are resolved and that the testing described in this procedure can be completed.

1.2.2 The MPS Specialist shall be responsible for performing the tests required in this procedure and coordinating the efforts of subsystem specialists that are required to assist in operating the components of the MPS including the laser system, the high-voltage power supplies, and the instrumentation systems.

\subsection{Prerequisites}

NOTE:

See Attachment 1.8.1, MPS Configuration Checklist. 


\subsubsection{The logic of the MPS controller was pre-checked and debugged. The controller is}

connected to the controls network.

\subsubsection{The MPS-to-laser interface has been established. The MPS controller has a direct link} to:

a. The laser PC controller.

b. The laser shutter controller

c. The intensity control EOM (not ready by start of the tests)

\subsubsection{The MPS-to-gun interface has been established:}

a. The MPS controller obtains the readings of the gun HVPS status (on/off status, fault status and voltage readings).

b. The MPS has a direct link to gun protection system that allows MPS controller to turn off the gun HVPS.

c. The MPS controller is receiving the gun vacuum readings.

\subsubsection{The MPS diagnostic subsystems have been installed, tested, and connected to the} MPS controller:

a. The FCT and the PD have been installed, tested, and connected to the MPS and to the network.

- Based on the bench test calibration, the MPS/FCT specialist entered the Q5 values corresponding to zero level (no current), IUSOM and $10 \mathrm{~kW}$ dump level.

b. The BLMs have been installed tested and connected to the MPS and to the network.

- The best guess for the BLM signal level corresponding to the start-up lloss value has been provided by the diagnostic group.

c. Vacuum gauges have been installed and tested.

- The integral "good/bad" vacuum signal has been provided to the MPS controller.

d. The calculation of the FCT/PD differential signal has been implemented.

- The "above/below" FCT/PD differential signal corresponding to the threshold of $80 \square$ A beam current has been provided to the MPS controller.

e. BPMs have been installed and tested.

- The initial setting for the tolerable trajectory window has been provided to the instrumentation group by the cognizant accelerator physicist (initial window setting of $+/-5 \mathrm{~mm}$ is recommended).

- The sum signal for "trajectory is inside/outside window" has been provided to the MPS controller.

f. Magnets (lecs1-inj.d1-ps, lecs1-gun.th1-ps and lecs1-gun.tv1-ps) have been installed, connected to respective PS, calibrated and tested.

- Magnet PS readings ("on/off" status and nominal setting with +/- 1\% range for lecs1-inj.d1-ps, and nominal settings with $+/-10 \%$ range for lecs1-gun.th1-ps and lecs1-gun.tv1-ps) have been provided to the MPS controller.

- Magnets PS are connected to the network.

g. Insertion devices are installed, tested, and connected to the MPS and to the network.

\subsection{Precautions}

\section{NONE}

\subsection{Procedure}




\subsubsection{Fail-Safe Test of Integrated System}

NOTE:

Some of these tests might have been already completed when respective signals were provided to the controller.

IF any of the tests listed below fail, THEN the MPS specialist may proceed with remaining fail-safe tests but further system commissioning must be halted until the issue is investigated and resolved.

\section{Initial System State:}

- The laser shutter is open.

- The gun HVPS is set to zero and disabled.

- The laser intensity is set to zero.

\subsubsection{Check the fail-safe setup for MPS-to-laser interface.}

NOTE:

The test is performed from building 1002D by the MPS specialist.

The operation of the laser shutter is monitored in building $1002 \mathrm{~F}$ by the laser specialist.

a. Disconnect the MPS-to-PC cable, observe the PC closing. Reconnect the Pockels Cell link.

b. Disconnect the MPS-to-shutter cable, observe the shutter closing. Reconnect the shutter cable. Open laser shutter.

\subsubsection{Check the fail-safe setup of the MPS-to-gun interface.}

\section{NOTE:}

The test is performed from building $1002 \mathrm{D}$ by the MPS and the gun specialists.

a. Disconnect the HVPS status link from the MPS controller and observe the laser shutter closing. Reconnect the HVPS link and open the shutter.

b. Disconnect the HVPS readback from the MPS controller and observe the laser shutter closing. Reconnect the HVPS readback and open the shutter.

c. Disconnect the gun vacuum link from the MPS controller and observe the laser shutter closing. Reconnect the HVPS link and open the shutter.

\section{NOTE:}

Tests 1.5.1.3 - 1.5.1.21 are performed from building 1002D by the MPS specialist.

\subsubsection{Check the fail-safe setup of the FCT/PD current level measurement links.}

Disconnect the FCT current level measurement link from the MPS controller and observe the laser shutter closing. Reconnect the link and open the laser shutter. Repeat for the PD current level measurement link. 


\subsubsection{Emulate the beam current level IUSOM.}

\subsubsection{Check the fail-safe setup of the HVPS voltage in-range link.}

Disconnect the DC Gun HVPS voltage link from the MPS controller and observe the laser shutter closing. Reconnect the cable and open the shutter.

\subsubsection{Check the fail-safe setup of the HVPS fault status link.}

Disconnect the DC Gun HVPS fault status link from the MPS controller and observe the laser shutter closing. Reconnect the cable and open the shutter.

\subsubsection{Check the fail-safe setup of the BLM link.}

Disconnect the BLM signal from the MPS controller and observe the laser shutter closing. Reconnect the cable and open the shutter.

\subsubsection{Check the fail-safe setup of the vacuum link.}

Disconnect the vacuum signal from the MPS controller and observe the laser shutter closing. Reconnect the cable and open the shutter.

\subsubsection{Check the fail-safe setup of the FCT/PD differential link.}

Disconnect the FCT/Laser Differential link from the MPS controller and observe the laser shutter closing. Reconnect the cable and open the shutter.

\subsubsection{Emulate the beam current level corresponding to level 1 (FC Limit).}

\subsubsection{Repeat steps 1.5.1.5 through 1.5.1.9 for this current level.}

\subsubsection{Check the fail-safe setup of the magnets link.}

Disconnect the Gun Corrector PS Current signal from the MPS controller and observe the laser shutter closing. Reconnect the cable and open the shutter.

\subsubsection{Check the fail-safe setup of the vacuum valves link.}

Disconnect the vacuum valves signal from the MPS controller and observe the laser shutter closing. Reconnect the cable and open the shutter.

\subsubsection{Check the fail-safe setup of the insertion devices link.}

Disconnect the insertion devices signal from the MPS controller and observe the laser shutter closing. Reconnect the cable and open the shutter.

\subsubsection{Check the fail-safe setup of the FC temperature link.}


Disconnect the FC temperature signal from the MPS controller and observe the laser shutter closing. Reconnect the cable and open the shutter.

\subsubsection{Emulate the beam current level corresponding to level 2 (Dump Limit).}

\subsubsection{Repeat steps 1.5.1.11 through 1.5.1.14 for this current level.}

\subsubsection{Check the fail-safe setup of the BPM link.}

Disconnect the BPM signal from the MPS controller and observe the laser shutter closing. Reconnect the cable and open the shutter.

\subsubsection{Check the fail-safe setup of the dipole link.}

Disconnect the Bending Magnet Current signal from the MPS controller and observe the laser shutter closing. Reconnect the cable and open the shutter.

\subsubsection{Check the fail-safe setup of the FCT differential link.}

Disconnect the FCT Differential signal from the MPS controller and observe the laser shutter closing. Reconnect the cable and open the shutter.

\subsubsection{Check the fail-safe setup of the water flow link.}

Disconnect the Water signal from the MPS controller and observe the laser shutter closing. Reconnect the cable and open the shutter.

\subsubsection{Check the fail-safe setup of the dump line temperature link.}

Disconnect the Dump Line Temperature signal from the MPS controller and observe the laser shutter closing. Reconnect the cable and open the shutter.

\subsubsection{Test of Integrated System Logic}

NOTE:

If any of the tests listed below fail, THEN the MPS Specialist may proceed with remaining logic tests but farther system commissioning must be halted until the issue is investigated and resolved.

Use Attachment 2.8.1, Integrated MPS Test Result Checklist to record final test results.

\section{Assumptions:}

- Alignment laser is passed to and is observable on the cathode (or the blank pluck).

- The current level is emulated either by adjusting levels in the FCT controller or by an external generator connected to the MPS controller via the current level link. 
- The laser interlock means that the Pockels cell and the laser shutter are getting closed. It must cause the disappearance of the laser on the cathode.

\section{Initial System State:}

- The laser shutter is closed.

- The gun HVPS is set to zero and the HVPS is disabled.

- The laser intensity is set to zero and the emulated beam current level is set to zero level.

NOTE:

All tests listed below are performed from building 1002D by the MPS Specialist.

\subsubsection{Check the system protection for isolation and laser alignment modes.}

a. Open the laser shutter, enable the HVPS and observe the laser interlock.

b. Set gun HVPS to $400 \mathrm{kV}$, open the laser shutter, drop gun voltage to $350 \mathrm{kV}$ and observe the interlock.

\subsubsection{Check system protection for the USOM level.}

a. Set gun voltage to $400 \mathrm{kV}$ and open the laser shutter.

b. Emulate the beam current level at IUSOM.

c. Reduce the gun HV to $300 \mathrm{kV}$ and observe the laser interlock. Set HVPS to $400 \mathrm{kV}$ and open the laser shutter.

d. Switch off the controller for one of the vacuum gauges monitored by the MPS and observe the laser interlock. Turn on the controller and open the laser shutter.

\subsubsection{Check system protection for the FC level.}

a. Turn lecs1-inj.d1-ps (dipole) off.

b. Set lecs1-gun.th1-ps and lecs1-gun.tv1-ps to their nominal values.

c. Emulate the beam current level above IUSOM but below IFC.

d. Insert the gun profile monitor (lecs-1-inj.yag1) and observe the interlock. Remove the profile monitor and open the laser shutter.

e. Repeat step (d) for each and every insertion device (all vacuum valves, slit, lecs-1-inj.yag0, 2 \& 3).

f. Change lecs1-gun.th1-ps setting by $15 \%$ and observe the interlock. Set lecs1-gun.th1-ps to its nominal value and open the laser shutter.

g. Repeat step (f) for lecs1-gun.tv1-ps.

h. Emulate the beam current level exceeding IFC and observe the interlock. Return emulated current to the previous value and open the laser shutter.

i. Reduce the gun HV to $300 \mathrm{kV}$ and observe the laser interlock. Set HVPS to $400 \mathrm{kV}$ and open the laser shutter.

j. Switch off the controller for one of the vacuum gauges monitored by the MPS and observe the laser interlock. Turn on the controller and open the laser shutter.

k. On the FC temperature controller reduce the temperature threshold below the currently measured temperature and observe the laser interlock. Return temperature threshold to its nominal value and open the laser shutter. 


\subsubsection{Check system protection for the BD level.}

a. Set gun voltage to $400 \mathrm{kV}$ and open the laser shutter.

b. Turn lecs1-inj.d1-ps (dipole) on and set its current to the nominal value.

c. Set lecs1-gun.th1-ps and lecs1-gun.tv1-ps to their nominal values.

d. Emulate the beam current level above IUSOM but below IBD.

e. Insert the gun profile monitor (lecs-1-inj.yag1) and observe the interlock. Remove the profile monitor and open the laser shutter.

f. Repeat step (e) for each and every insertion device (all vacuum valves, slit, lecs-1-inj.yag0, 2 \& 3).

g. Turn off lecs1-inj.d1-ps (dipole) and observe the interlock. Turn lecs1-inj.d1-ps (dipole) back on, make sure it is at nominal setting and open the laser shutter.

h. Change lecs1-gun.th1-ps setting by $15 \%$ and observe the interlock. Set lecs1-gun.th1-ps to its nominal value and open the laser shutter.

i. Repeat step (h) for lecs1-gun.tv1-ps.

j. Emulate the beam current level exceeding IBD and observe the interlock. Return emulated current to the previous value and open the laser shutter.

k. Reduce the gun HV to $300 \mathrm{kV}$ and observe the laser interlock. Set HVPS to $400 \mathrm{kV}$ and open the laser shutter.

I. Switch off the controller for one of the vacuum gauges monitored by the MPS and observe the laser interlock. Turn on the controller and open the laser shutter.

$\mathrm{m}$. On the FC temperature controller reduce the temperature threshold below the currently measured temperature and observe the laser interlock. Return temperature threshold to its nominal value and open the laser shutter.

$\mathrm{n}$. Switch off the water flow to the BD and observe the laser interlock. Switch on the water flow and open the laser shutter.

\subsection{Documentation}

\section{NONE}

\subsection{References}

\section{NONE}

\subsection{Attachments}

\section{Attachment 1.8.1}

MPS Configuration Checklist (for 2017)

\begin{tabular}{|l|l|}
\hline MPS controller is installed in crate in 1002D MPS controller is connected to the network & \\
\hline Laser PC/PC controller has been tested & \\
\hline Laser PC controller is connected to the MPS controller & \\
\hline Laser shutter has been tested & \\
\hline Laser shutter is connected to the MPS controller & \\
\hline Gun HVPS on/off status is provided to the MPS controller & \\
\hline
\end{tabular}




\begin{tabular}{|l|l|}
\hline Gun voltage readings are provided to the MPS controller & \\
\hline Gun vacuum readings are provided to the MPS controller & \\
\hline MPS controller "gun shutdown" output is provided to the gun protection system & \\
\hline FCT has been tested and installed in the tunnel & \\
\hline PD has been tested and installed in the laser trailer & \\
\hline FCT and PD controllers have been tested, installed in 1002D and connected to the FCT and PD & \\
\hline $\begin{array}{l}\text { Q5 values for zero level (no current), IUSOM and 10 kW dump level were identified and } \\
\text { programmed in the FCT/PD controller }\end{array}$ & \\
\hline FCT/PD controller is connected to the MPS controller & \\
\hline BLMs have been installed in the tunnel & \\
\hline BLMs electronics have been tested, installed in 1002D and connected to BLMs & \\
\hline BLM electronics is providing input to the MPS controller & \\
\hline Vacuum gauges are installed in the tunnel & \\
\hline The vacuum gauges are connected to the electronics in 1002D & \\
\hline The "good/bad" vacuum summary signal is provided to the MPS controller & \\
\hline BPMs have been installed in the tunnel & \\
\hline BPMs electronics is installed in 1002D and connected to the BPMs & \\
\hline $\begin{array}{l}\text { The sum signal for "trajectory is inside/outside window" has been provided to the MPS } \\
\text { controller }\end{array}$ & \\
\hline $\begin{array}{l}\text { Magnets (lecs1-inj.d1-ps, lecs1-gun.th1-ps and lecs1-gun.tv1-ps) have been installed in the } \\
\text { tunnel }\end{array}$ & \\
\hline $\begin{array}{l}\text { Magnets (lecs1-inj.d1-ps, lecs1-gun.th1-ps and lecs1-gun.tv1-ps) PS have been installed in } \\
\text { 1002D and connected to the magnets }\end{array}$ & \\
\hline $\begin{array}{l}\text { Magnets (lecs1-inj.d1-ps, lecs1-gun.th1-ps and lecs1-gun.tv1-ps) PS “on/off" status is } \\
\text { provided to the MPS controller }\end{array}$ & \\
\hline $\begin{array}{l}\text { Magnets (lecs1-inj.d1-ps, lecs1-gun.th1-ps and lecs1-gun.tv1-ps) PS “in/outside allowed } \\
\text { window" status is provided to the MPS controller }\end{array}$ & \\
\hline Vacuum valves are installed in the tunnel & \\
\hline Valves controllers are installed in 1002 D and connected to the valves & \\
\hline The summary signal of valves "in/out" status has been provided to the MPS controller & \\
\hline $\begin{array}{l}\text { Insertion devices (lecs-1-inj.yag0, 1, 2 \& 3 and emittance slit) have been installed in the } \\
\text { tunnel }\end{array}$ & \\
\hline Insertion devices are connected to the controllers in 1002D & \\
\hline Insertion devices “in/out" status is provided to the MPS controller & \\
\hline
\end{tabular}




\section{Attachment 1.8.2}

\section{Integrated MPS Test Result Checklist}

\begin{tabular}{|l|l|}
\hline MPS is certified as a fail-safe system (tests 1.5.1 were performed successfully) & \\
\hline $\begin{array}{l}\text { The logic of isolation and laser alignment modes is checked (test 1.5.2.1 was } \\
\text { successfully performed) }\end{array}$ & \\
\hline $\begin{array}{l}\text { The system protection for the USOM current level is verified (tests 1.5.2.2 were } \\
\text { successfully passed) }\end{array}$ & \\
\hline $\begin{array}{l}\text { The system protection for the FC current level is verified (tests 1.5.2.3 were } \\
\text { successfully passed) }\end{array}$ & \\
\hline $\begin{array}{l}\text { The system protection for the BD current level is verified (tests 1.5.2.4 were } \\
\text { successfully passed) }\end{array}$ & \\
\hline $\begin{array}{l}\text { The protection against increasing beam power above } 10 \mathrm{~kW} \text { is verified (test 1.5.2.4 } \mathrm{j} \\
\text { was successfully passed) }\end{array}$ & \\
\hline
\end{tabular}




\subsection{MPS commissioning with beam (for 2017 run)}

\subsection{Purpose}

2.1.1 The purpose of this section is to commission both the components of the LEReC MPS and the entire integrated LEReC MPS with beam.

\subsubsection{Abbreviations and Symbols}

$\mathrm{BD}-10 \mathrm{~kW}$ beam dump

BLM - beam loss monitor

BPM - beam position monitor

EOM - electro-optical modulator

FCT - fast current transformer

HVPS - high-voltage power supply

MPS - machine protection system

PC - laser Pockels Cell

PD - laser photodiode installed on the laser table in the tunnel

PS - power supply

Q5 - beam charge accumulated in $5 \mathrm{~s}$ window (the measure of the beam current)

$\mathrm{QE}$ - quantum efficiency

USOM - ultimately safe operation mode

IBD - allowed current threshold for the beam delivered to the beam dump

lloss - threshold of the tolerable routine losses

IUSOM - current threshold for the ultimately safe operation mode

Ptrip - vacuum gauge reading corresponding to the beam losses that interlock the machine via vacuum trip

PUSOM - pressure reading of the vacuum gauge at loss location at IUSOM beam current

\subsection{Responsibilities}

2.2.1 The LEReC Liaison Physicist is responsible for ensuring that the integrated system test is completed and that the MPS system is correctly configured. Problems with components of the system or issues that arise during testing should be reported to the Liaison Physicist as soon as possible to ensure that these issues are resolved and that the testing described in this procedure can be completed.

2.2.2 The MPS Specialist shall be responsible for performing the tests required in this procedure and coordinating the efforts of subsystem specialists that are required to assist in operating the components of the MPS including the laser system, the high- voltage power supplies, and the instrumentation systems.

\subsection{Prerequisites}


2.3.1 The MPS successfully passed at minimum the following steps of the integrated system test without beam: 1.5.1.1 - 1.5.1.4, and 1.5.2.1.

2.3.2 The LEReC is ready for the work with the beam.

\subsection{Precautions}

NOTE:

These tests must be completed without masking any inputs unless the permission for masking of the particular input is given by the project manager.

\subsection{Procedure}

\subsubsection{Working in the USOM and Checking Calibration of FCT/PD System}

Initial State of System:

- The laser is set to provide the LCM beam parameters.

- The laser shutter is closed.

- The HVPS is set to $400 \mathrm{kV}$.

\subsubsection{Check the MPS USOM operation.}

a. Open the laser shutter.

b. Check that the FCT readings give the MPS controller the proper beam current level (above 0 current and bellow IUSOM).

c. Measure QE and adjust PD to MPS controller output accordingly. Check that the PD readings give the MPS controller the proper beam current level (above 0 current and bellow IUSOM).

d. Increase bunch charge to $200 \mathrm{pC}$.

e. Check that FCT/PD measured Q5 $=30 \mathrm{nC}$.

f. Check that FCT/PD differential signal is showing $\mathrm{Q} 5 \lesssim 0.3 \mathrm{nC}$.

g. Increase laser pulse length to 6 macrobunches.

h. Check that FCT/PD measured Q5 $=180 \mathrm{nC}$.

i. Check that FCT/PD differential signal is showing $\mathrm{Q} 5 \lesssim 18 \mathrm{nC}$.

j. Based on the measurement in step (h) adjust IUSOM level so that respective Q5 is $200 \mathrm{nC}$ (equivalent to IUSOM $=40 \mathrm{nA}$ ).

k. Insert the first (gun) profile monitor.

I. Increase the laser pulse length to 7 macrobunches and observe the interlock.

$\mathrm{m}$. Set laser to LCM parameters.

n. Open laser shutter.

\subsubsection{Check MPS logic with the beam.}


a. Transport beam to the BD.

b. When you are satisfied with beam trajectory take notice of lecs1-inj.d1-ps and lecs1-gun.th1-ps and lecs1-gun.tv1-ps settings - these are your nominal magnet settings for the MPS controller to be locked to.

c. Make notice of the BPM readings - these are your nominal BPM readings for the MPS controller to be locked to.

d. Set the USOM level for FCT/PD signal to the controller to $Q 5=40 \mathrm{nC}$.

e. Set bunch charge to $200 \mathrm{pC}$ and set laser pulse length to 2 macrobunches.

f. Check that MPS reads the beam current level above USOM and below IBD.

g. Insert the gun profile monitor (lecs-1-inj.yag1) and observe the interlock.

h. Remove the gun profile monitor and open the laser shutter.

i. Repeat steps (g-h) for each and every insertion device (all vacuum valves, emittance slit, lecs-1inj.yag0, 2 \& 3).

j. Turn off lecs1-inj.d1-ps (dipole) PS and observe the interlock.

k. Turn on lecs1-inj.d1-ps (dipole) and set it to the nominal current, then open the laser shutter.

I. Set lecs1-inj.d1-ps (dipole) current to half of its nominal value and observe the interlock.

m. Set lecs1-inj.d1-ps (dipole) to its nominal value and open the laser shutter.

n. Change lecs1-gun.th1-ps setting by $15 \%$ and observe the interlock.

o. Set lecs1-gun.th1-ps PS current to its nominal value and open the laser shutter

p. Repeat steps ( $\mathrm{n}-\mathrm{o}$ ) for lecs1-gun.tv1-ps.

q. Using correctors other than lecs1-gun.th1-ps and lecs1-gun.tv1-psmove beam trajectory outside of allowed BPM window and observe the beam interlock.

$r$. Return the settings of correctors used in step $(q)$ to their initial values and open the laser shutter.

s. Set the IBD threshold to $70 \mathrm{nC}$.

t. Set laser pulse length to 3 macrobunches and observe the interlock.

u. Return IUSOM and IBD thresholds to their nominal values and open the laser shutter.

v. Set Q5 for FCT/PD threshold to $30 \mathrm{nC}$.

w. Insert the gun profile monitor and observe the interlock.

$x$. Return FCT/PD threshold to its nominal value, remove gun profile monitor and open the laser shutter.

y. At this point, the MPS is set back to its design configuration; the test of the system logic is complete (with the exception of BLMs and vacuum); the beam current shall be below design IUSOM and any beam/insertion device manipulations shall not cause machine interlock.

\subsubsection{Check MPS interaction with vacuum gauges.}

a. Start with LCM beam settings.

b. By steering the beam trajectory create the loss point near one of the vacuum gauges.

c. By increasing the beam current (increase charge/bunch and number of macrobunches) up to IUSOM increase the pressure readings from the gauge of your choice. 
d. If at some point the observed pressure becomes larger than the design vacuum threshold (Ptrip - defined by the vacuum group) then you shall observe the interlock.

e. If conditions of step (d) are satisfied then set the trajectory correctors back to their nominal values, wait for the pressure to get back to its normal level and open the shutter. Your MPS-vacuum tests are complete. Otherwise, go through the steps $(f-j)$.

f. If the conditions of step (d) are not satisfied then notice the pressure at IUSOM and call this pressure PUSOM.

g. Reset beam settings to the LCM ones.

h. Set vacuum pressure threshold (Ptrip) to PUSOM/2.

i. Increase beam current to such current ( $\gtrsim I U S O M / 2$ ) that the readings of the gauge at the loss location exceed PUSOM/2 and observe the interlock.

j. Set Ptrip back to its nominal value, set trajectory correctors back to their nominal value and open the shutter. MPS-vacuum tests are complete.

\subsubsection{Tolerable Beam Loss Studies, BLM Calibration and Check of MPS-BLM Logic}

\section{NOTE:}

In these tests, the beam losses are intentionally created at beam current exceeding initial setting for the threshold of tolerable loss current (initial lloss). The beam current can be as high as but not higher than Imax (corresponding Q5 = $3 \mathrm{mC}$ ). In steps (a-j), the losses must be created by beam defocusing only and not by steering the beam trajectory so that the vacuum chamber experiences direct hit or by inserting the in-vacuum devices. These studies must be reviewed and pre-approved by the LEReC Liaison Physicist. The studies must be performed by the MPS accelerator physics coordinator. The tests must be performed in the presence of the BLM subsystem specialist and MPS specialist.

a. Set the beam settings to the LCM ones.

b. Transport beam to the dump.

c. Check that all the insertion devices are out.

d. Check that the BPM readings are within the predefined window.

e. Check that the magnets lecs1-inj.d1-ps, lecs1-gun.th1-ps and lecs1-gun.tv1-ps are at the nominal settings.

f. Defocus the beam so that the distributed beam losses are created at such location that the BLM at the loss location is not picking up the noise from the beam dump but the loss is happening as far from the gun as possible.

g. Set the USOM level for FCT/PD to Q5 = $3 \mathrm{mC}$, which corresponds to Imax. It is essential that from this point on and until the step (j) neither insertion devices nor the trajectory correctors are manipulated. The operator shall be aware of the vacuum readings and beam trajectory all the time throughout the studies. If operator notices essential drift of beam trajectory (due to corrector malfunction) then the studies must be stopped (close the laser shutter and set USOM level for FCT/PD back to its nominal value).

h. Gradually increase the beam current (up to Imax if needed) and notice the current at which the vacuum trip is caused by the gauge near the loss location. Notice the BLM reading at the time of the trip. 
i. If the trip in step (h) never happened then the new Iloss $=2 / 3$ Imax $=400 \mu \mathrm{A}$ (equivalent Q5 $=2$ $m C)$, if the trip happens then the new lloss is equal to $2 / 3$ of the trip current.

j. Set IUSOM back to its design value and set beam focusing to the nominal "transport to BD" settings.

k. Set bunch charge to $200 \mathrm{pC}$ and set laser pulse length to 6 macrobunches.

I. Steer trajectory to the vacuum chamber wall near the BLM of your choice and notice the BLM readings

$\mathrm{m}$. The nominal BLM trip threshold must be set to the smaller of the signal observed in step (I) and signal corresponding to lloss found in step (i)

n. Set beam to the LCM.

o. Set BLM trip threshold to the signal observed from loss of the beam Q5 $=40 \mathrm{nC}$ on the profile monitor (lecs-1-inj.yag2).

p. Set bunch charge to $200 \mathrm{pC}$ and set laser pulse length to 2 macrobunches

q. Insert lecs-1-inj.yag2 and observe the BLM-caused interlock.

r. Remove the lecs-1-inj.yag2, reset BLM threshold to its nominal value and open the shutter.

s. At this point, the MPS is set back to its design configuration; the BLM signals are calibrated and the test of the MPS-BLM logic is complete; the beam current shall be below design IUSOM and any beam/insertion device manipulations shall not cause machine interlock.

\subsection{Documentation}

NONE

\subsection{References}

NONE

\subsection{Attachments}

NONE 


\section{Operation procedures}

Our experience shows that the procedures for routine MPS operation and check-ups must be based on the hands-on experience acquired during MPS/accelerator commissioning. It is essential that the routine operation procedures are written after the commissioning of the full MPS system is performed. Until then we suggest performing once a week a simplified MPS logic test consisting of the steps outlined in Section 9.1 below. We also present a first draft of the MPS test that shall be performed after any changes done to the MPS configuration.

\subsection{Routine MPS test}

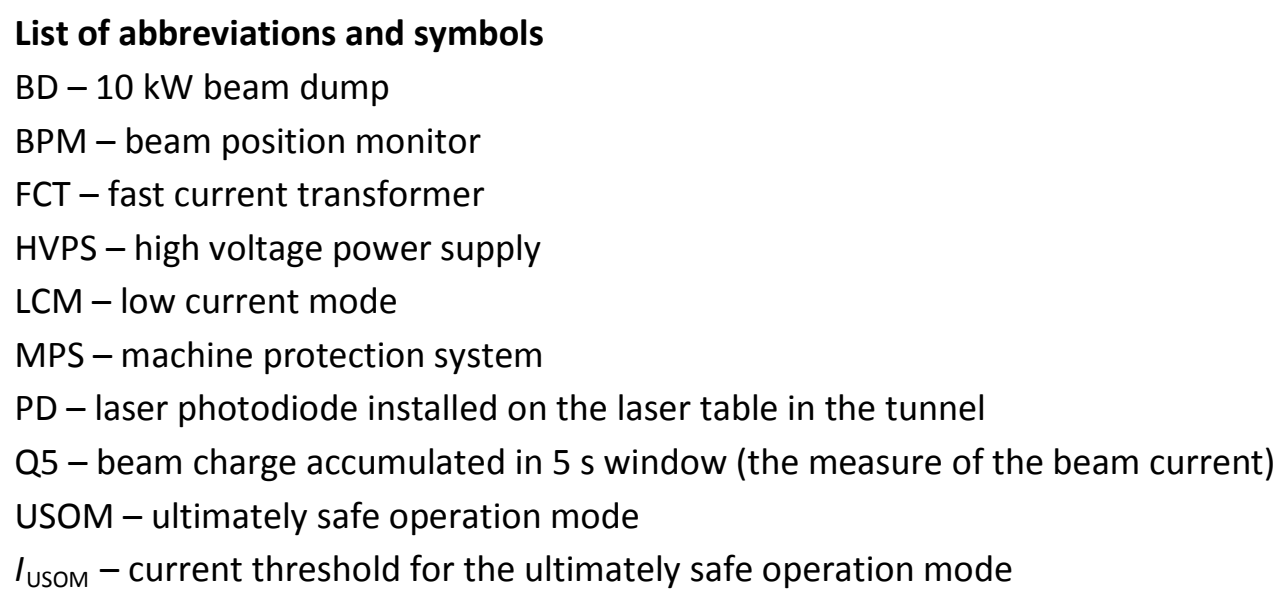

\section{The goal}

The goal of this procedure to perform a routine test of the LEReC MPS functionality.

\section{Check MPS logic with beam}

Initial state of the system:

The laser is set to provide the LCM beam parameters. The HVPS is set to $400 \mathrm{kV}$.

\subsection{Transport beam to the BD}

\subsection{Check MPS logic}

a. Set the USOM level for FCT/PD signal to the controller to $Q 5=40 \mathrm{nC}$

b. Set bunch charge to $200 \mathrm{pC}$ and set laser pulse length to 2 macrobunches

c. Insert the gun profile monitor and observe the interlock

d. Remove the gun profile monitor and open the laser shutter

e. Using correctors other than Corr24H/V and Corr25H/V move beam trajectory outside of allowed BPM window and observe the beam dump shutter

f. Return the settings of correctors used in step (q) to their initial values and open the laser

g. Return $I_{\text {USom }}$ threshold to its nominal value and open the laser shutter

\subsection{Verification of MPS integrity}

\section{List of abbreviations and symbols}


$\mathrm{BD}-10 \mathrm{~kW}$ beam dump

BLM - beam loss monitor

BPM - beam position monitor

EOM - electro-optical modulator

FCT - fast current transformer

HVPS - high voltage power supply

LCM - low current mode

MPS - machine protection system

PC - laser Pockels Cell

PD - laser photodiode installed on the laser table in the tunnel

PS - power supply

Q5 - beam charge accumulated in $5 \mathrm{~s}$ window (the measure of the beam current)

$\mathrm{QE}$ - quantum efficiency

USOM - ultimately safe operation mode

$I_{\mathrm{BD}}$ - allowed current threshold for the beam delivered to the beam dump

$I_{\text {loss }}$ - threshold of the tolerable routine losses

$I_{\text {Usom }}$ - current threshold for the ultimately safe operation mode

$P_{\text {trip }}$-vacuum gauge reading corresponding to the beam losses that interlock the machine via vacuum trip

$P_{\text {Usom }}$ - pressure reading of the vacuum gauge at loss location at $I_{\text {Usom }}$ beam current

\section{The goal}

The goal of this procedure is to verify the integrity of the LEReC MPS after modifications to the system.

\section{Verifying MPS functionality}

Initial state of the system:

The laser is set to provide the LCM beam parameters. The laser shutter is closed. The HVPS is set to $400 \mathrm{kV}$.

\subsection{Check the MPS USOM operation}

a. Open the laser shutter

b. Check that the FCT readings give the MPS controller the proper beam current level (above 0 current and bellow / Usom)

c. Increase bunch charge to $200 \mathrm{pC}$

d. Check that FCT/PD measured Q5 $=30 \mathrm{nC}$

e. Check that FCT-PD differential signal is showing Q $5 \lesssim 0.3 \mathrm{nC}$

f. Increase laser pulse length to 6 macrobunches.

g. Check that FCT/PD measured Q5 $=180 \mathrm{nC}$

h. Check that FCT-PD differential signal is showing Q $5 \lesssim 18 \mathrm{nC}$

i. Insert the first (gun) profile monitor

j. Increase the laser pulse length to 7 macrobunches and observe the interlock

k. Set laser to LCM parameters

I. Open laser shutter 


\subsection{Check MPS logic with the beam}

a. Transport beam to the BD

b. Set the USOM level for FCT/PD signal to the controller to $Q 5=40 \mathrm{nC}$

c. Set bunch charge to $200 \mathrm{pC}$ and set laser pulse length to 2 macrobunches

d. Check that MPS reads the beam current level above USOM and below $I_{\mathrm{BD}}$

e. Insert the gun profile monitor (lecs-1-inj.yag1) and observe the interlock

f. Remove the gun profile monitor and open the laser shutter

g. Repeat steps (e-f) for each and every insertion device (all vacuum valves, emittance slit, lecs1-inj.yag0,2 \& 3)

h. Turn off the dipole PS and observe the interlock

i. Turn on the dipole PS and set it to the nominal current, then open the laser shutter

j. Set dipole PS current to half of its nominal value and observe the interlock

k. Set dipole PS to its nominal value and open the laser shutter

I. Change Corr $24 \mathrm{H}$ setting by $15 \%$ and observe the interlock

$\mathrm{m}$. Set Corr24H PS current to its nominal value and open the laser shutter

n. Repeat steps (I-m) for Corr24V and Corr25H/V

o. Using correctors other than Corr24H/V and Corr25H/V move beam trajectory outside of allowed BPM window and observe the beam dump

p. Return the settings of correctors used in step (o) to their initial values and open the laser shutter

q. Set the $I_{\mathrm{BD}}$ threshold to $70 \mathrm{nC}$

r. Set laser pulse length to 3 macro-bunches and observe the interlock

s. Return $I_{\text {UsOM }}$ and $I_{\mathrm{BD}}$ thresholds to their nominal values and open the laser shutter

t. Set Q5 for FCT-PD threshold to $30 \mathrm{nC}$

$u$. Insert the gun profile monitor and observe the interlock

v. Return FCT-PD threshold to its nominal value, remove gun profile monitor and open the laser shutter.

w. Set BLM trip threshold to the signal observed from loss of the beam $Q 5=40 \mathrm{nC}$ on the profile monitor lecs-1-inj.yag2

$\mathrm{x}$. Set bunch charge to $200 \mathrm{pC}$ and set laser pulse length to 2 macrobunches

$y$. Insert lecs-1-inj.yag2 and observe the BLM-caused interlock

z. Remove the lecs-1-inj.yag2, reset BLM threshold to its nominal value and open the shutter A this point the MPS is set back to its design configuration; the test of the system logic is complete; the beam current shall be below design $I_{\text {usom }}$ and any beam/insertion device manipulations shall not cause machine interlock. 


\section{Conclusion}

We described the LEReC machine protection system. In conclusion we repeat the most important results of our work.

The basic system parameters were derived in Section 2 and were summarized in Table 4 (repeated below).

\begin{tabular}{|c|c|c|c|}
\hline \multicolumn{4}{|c|}{ LEReC MPS parameters } \\
\hline Parameter & Symbol & Value & Comments \\
\hline Reaction time & $\mathrm{t}_{\text {react }}$ & 20 us & $\begin{array}{l}\text { Derived under assumption that beam trajectory and } \\
\text { focusing studies are performed in LCM only and that } \\
\text { in HCM the beam trajectory is locked in some BPMs } \\
\text { and some magnet power supply currents are locked } \\
\text { at operational value. }\end{array}$ \\
\hline Tolerable routine losses & $\mathrm{I}_{\text {loss }}$ & $1 \mathrm{uA}$ & $\begin{array}{l}\text { The eventual setting for tolerable loss threshold will } \\
\text { be found experimentally. The beam current used in } \\
\text { such studies must not exceed } 600 \text { uA. }\end{array}$ \\
\hline $\begin{array}{l}\text { Ultimately safe } \\
\text { operation mode } \\
\text { threshold }\end{array}$ & $\mathrm{I}_{\text {USOM }}$ & $40 \mathrm{nA}$ & $\begin{array}{l}\text { The LCM is the USOM. } \\
\text { The TM1 is the USOM if } \mathrm{N}_{\mathrm{mb}} \leq 6\end{array}$ \\
\hline
\end{tabular}

Table 4 (repeated): Basic MPS parameters

The failure scenarios and resulting requirements to the MPS diagnostics were considered in Sections 3 and 4. Various FCT readings corresponding to the current levels important to the MPS are listed in Table 6 (repeated below).

\begin{tabular}{|c|c|c|c|c|}
\hline Beam mode & Machine mode & current & $\begin{array}{l}\text { Q accumulated in } \\
5 \text { s long window }\end{array}$ & Comments \\
\hline LCM & Flags, valves, etc. & $6 \mathrm{nA}$ & $30 \mathrm{nC}$ & \\
\hline \multirow[t]{2}{*}{ USOM } & Flags, valves, etc. & $40 \mathrm{nA}$ & $200 \mathrm{nC}$ & \\
\hline & & $1 \mathrm{uA}$ & 5 uC & Initial I loss \\
\hline \multirow[t]{4}{*}{ RFSM } & RF line flag/dump & 3 uA & $15 \mathrm{uC}$ & \\
\hline & & 80 uA & $400 \mathrm{uC}$ & FCT-PD threshold \\
\hline & & 600 uA & $3 \mathrm{mc}$ & $\begin{array}{l}\mathrm{I}_{\max }-\text { threshold for studies } \\
\text { of tolerable } \mathrm{I}_{\text {loss }} \text { and BLM } \\
\text { settings }\end{array}$ \\
\hline & $10 \mathrm{~kW}$ dump & $4 \mathrm{~mA}$ & $20 \mathrm{mC}$ & $\begin{array}{l}\text { current/charge are } \\
\text { calculated for } 2.6 \mathrm{MeV} \text { beam }\end{array}$ \\
\hline $\mathrm{TM}, \mathrm{HCM}$ & $140 \mathrm{~kW}$ dump & $55 \mathrm{~mA}$ & $275 \mathrm{mc}$ & \\
\hline CWM & $140 \mathrm{~kW}$ dump & $85 \mathrm{~mA}$ & $425 \mathrm{mc}$ & \\
\hline
\end{tabular}

Table 6 (repeated): Charge in $5 \mathrm{~s}$ window defines the MPS current levels. 
Detailed description of the MPS logic was presented in Section 7. The general schematic of the MPS system is presented in Fig. 21 (repeated below).

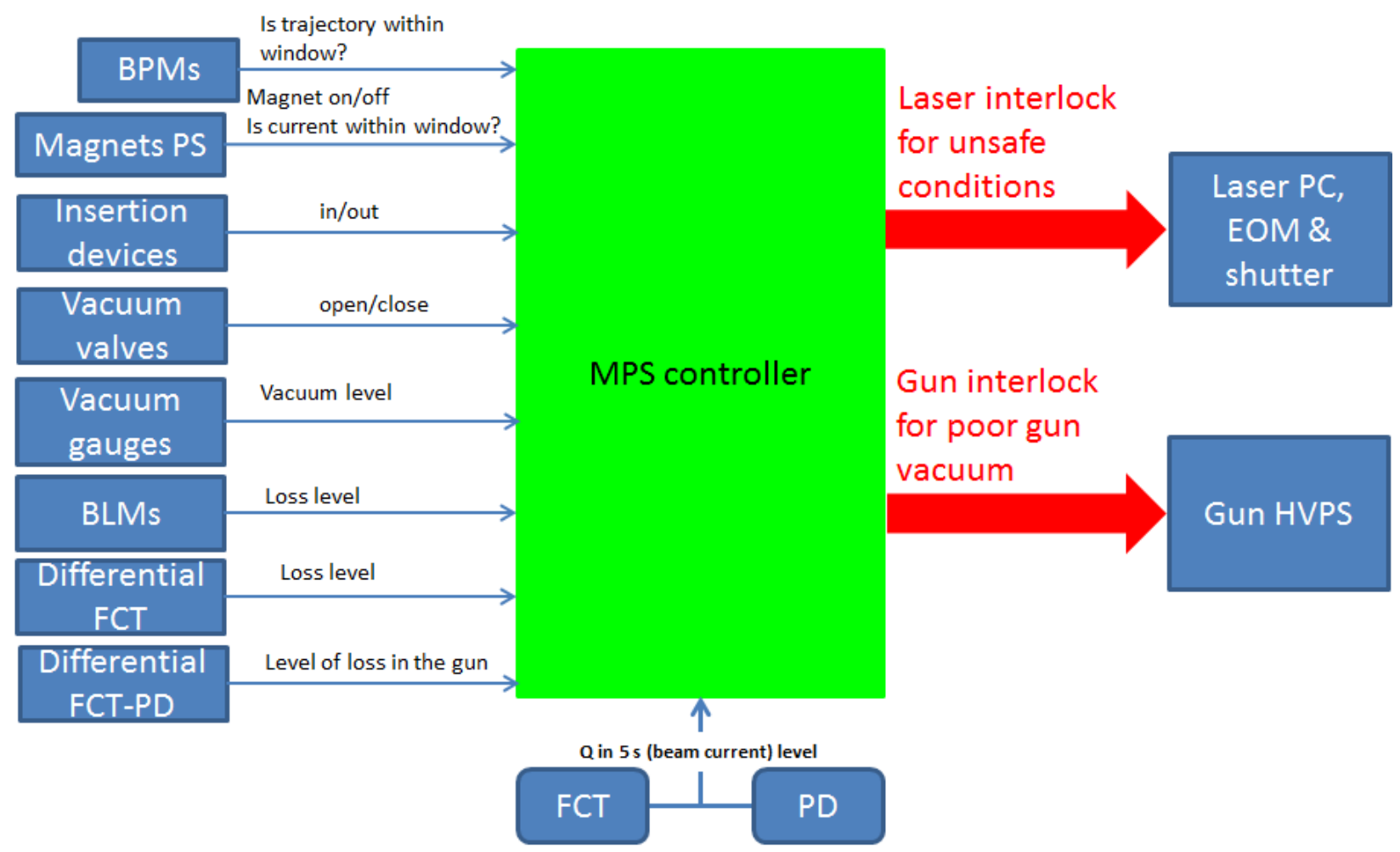

Figure 21 (repeated): Schematic of LEReC MPS

The schematic of the complete MPS logic for the gun test run is presented in Fig. 23 and Table 7 (repeated below). 


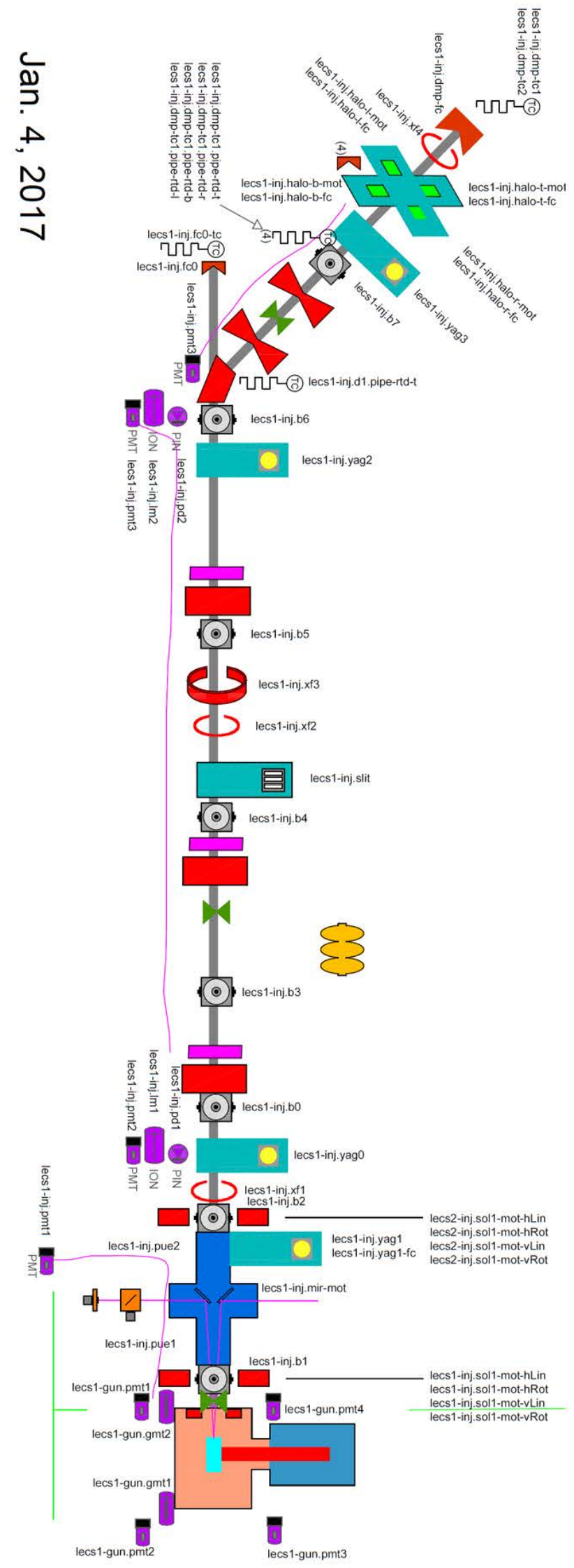




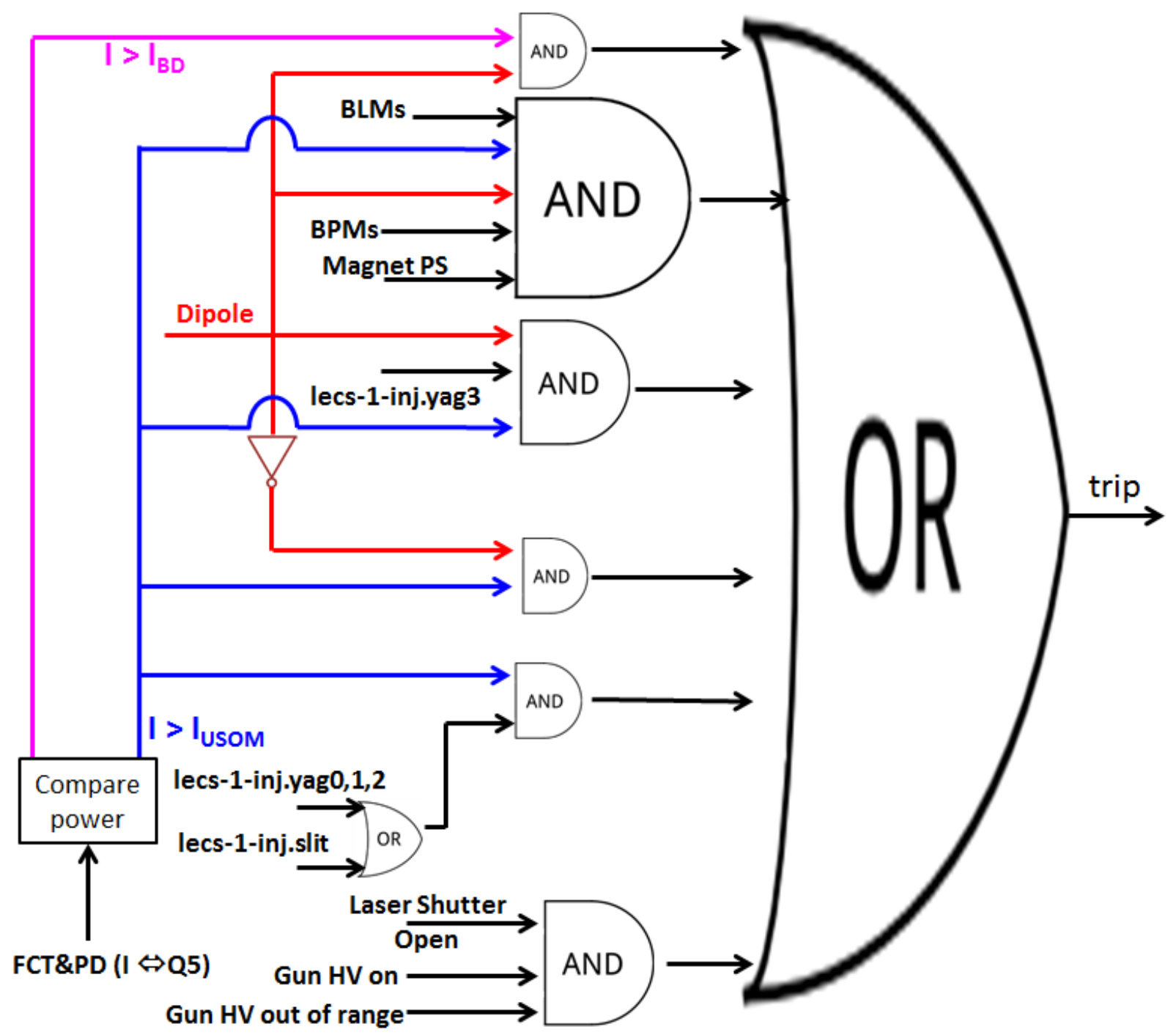

Figure 23 (repeated): Block diagram of the MPS logic for the gun test run. 


\section{0}

\begin{tabular}{|l|l|l|}
\hline Dipole & Off & On \\
\hline BPMs & In range & Not in range \\
\hline Magnet PS & In range & Not in range \\
\hline lecs-1-inj.yag3 & Out & In \\
\hline lecs-1-inj.yag0,1,2 & Out & In \\
\hline lecs-1-inj.slit & Out & In \\
\hline BLMs & $\begin{array}{l}\text { Losses are } \\
\text { below limit }\end{array}$ & $\begin{array}{l}\text { Losses are } \\
\text { above limit }\end{array}$ \\
\hline
\end{tabular}

Table 7 (repeated): The states of the MPS inputs on Fig. 23.

Finally, the detailed procedures for the MPS commissioning are given in Section 8. 